\title{
Electric Breakdown in Long Discharge Tubes at Low Pressure (Review)
}

\author{
Yu. Z. Ionikh* \\ St. Petersburg State University, St. Petersburg, 199034 Russia \\ *e-mail: y.ionikh@spbu.ru
}

Received March 19, 2020; revised April 9, 2020; accepted April 10, 2020

\begin{abstract}
The review is devoted to studies of the processes and mechanisms of ignition of a glow discharge in tubes whose length significantly exceeds their diameter (long discharge tubes) at low pressures ( $\sim 10$ Torr and lower) and moderate voltage rise rates $(\sim 1 \mathrm{kV} / \mu$ s and lower). The electric field in such tubes before a breakdown is substantially nonuniform. Therefore, a breakdown occurs after an ionization wave (or waves) passes through the discharge gap at a speed of $\sim 10^{5}-10^{7} \mathrm{~cm} / \mathrm{s}$. This makes the characteristics of the breakdown in long tubes significantly different from the breakdown between large and closely spaced electrodes, where the electric field is uniform before the breakdown and where the Townsend or, under strong overvoltage, streamer mechanism is realized. On the other hand, the nature of these processes is very different from those occurring in nanosecond discharges, which arise at voltages with a steepness of $\sim 1 \mathrm{kV} / \mathrm{ns}$ and higher and are associated with high-speed $\left(\sim 10^{9} \mathrm{~cm} / \mathrm{s}\right)$ ionization waves. The review is based on the materials of experimental and computational works published from 1938 to 2020. Breakdown processes, optical and electrical characteristics of the discharge gap during breakdown, and the influence of the external circuit parameters and external actions (shielding and illumination by external sources of visible radiation) are analyzed.
\end{abstract}

DOI: $10.1134 / \mathrm{S} 1063780 X 20100049$

\section{CONTENTS}

\section{Introduction}

2. Ionization waves

\subsection{Fast waves}

2.2. Pre-breakdown (slow) waves

3. Study of breakdown in long tubes under low pressure

3.1. Early works (until 1960)

3.2. Works of the 1960-1980s

3.3. Works of the 1990s and later

4. Manifestation of the wave nature of the breakdown upon discharge ignition in long tubes

4.1. Electrical signals in the discharge circuit

4.2. The effect of shielding on the breakdown

4.3. Breakdown voltage

4.4. "Memory effect" of the discharge gap

4.5. Initiation of breakdown by visible radiation

4.6. Breakdown in a tube with an ungrounded electrode

4.7. Radiation spectrum of the ionization wave

4.8. The discharge after the passage of the ionization wave

5. Conclusions

References

\section{INTRODUCTION}

1. Cylindrical discharge tubes with a length much greater than the diameter ("long" discharge tubes) began to be used in the $1850 \mathrm{~s}$ in the experiments of J. Plücker and H. Geissler at the University of Bonn [1]. Using a mercury pump created by Geissler, a stable reproducible low-pressure discharge in the tube was obtained, which was later called glow discharge. The term "Geissler" is used now for discharge tubes with a capillary insert (Fig. 1). Until recently, they were used as standard spectral sources. Plücker, experimenting with the created tubes, discovered cathode rays. In the subsequent works of J. Hittorf and W. Crookes, but under vacuum, the existence of the electron was proved (J.J. Thomson). For several decades, the glow-discharge plasma in a long tube, steady-state or decaying, has been widely used as a medium for studying elementary collision processes and transport characteristics of atoms and molecules with thermal energies [2]. This was facilitated by the presence in such a discharge of a region of a longitudinally uniform plasma: a positive column. For plasma physics, such studies provided information on the averaged energy and transport characteristics of electrons, on instabilities (contraction, stratification), etc. [3].

The practical application of long discharge tubes began with their use in outdoor advertising at the beginning of the 20th century ("neon signs"), which 


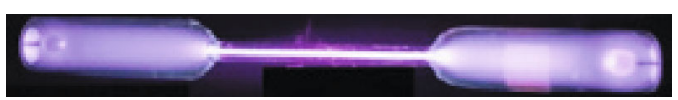

Fig. 1. Geissler tube.

reached its peak by the 1960s. At the beginning of the century, the first gas-discharge light source appeared, competing in a number of characteristics with the incandescent lamp: the Moore lamp (D.F. Moore). It was a discharge tube filled with carbon dioxide, whose radiation spectrum is close to that of natural light. Then began the development of a technology for the production of mercury fluorescent lamps, which gradually displaced incandescent lamps. The pinnacle of their evolution was compact energy-saving lamps with a long discharge tube coiled into a spiral, an electronic power supply circuit, and a standard lamp base. Currently, they are inferior in efficiency to LED light sources, but surpass them in spectral characteristics. A glow-discharge plasma in a long tube served as the active medium for the first continuous laser $(\mathrm{He}-\mathrm{Ne}$ mixture) [4]. This marked the beginning of the creation of an extensive class of gas-discharge lasers using various gases and their mixtures, as well as metal vapors. Although, at present, the field of practical application of many of them has narrowed due to the development of solid-state lasers, they nevertheless continue to be used in metrology, material processing, medicine, etc.

In a long tube, on applying a pulse of large amplitude and steepness, a so-called fast ionization wave arises: a potential gradient moving at a speed almost reaching the speed of light. Numerous scientific and technical applications of this phenomenon show promises in chemical technologies, for pumping laser media, and for generating high-energy electrons.

2. In many scientific and practical applications, a pulsed or pulse-periodic form of discharge is used. This raises the question of the processes of discharge ignition, i.e., electrical breakdown of the discharge gap. Breakdown phenomena were considered both in the earliest works devoted to discharge [5] and in later classical monographs [6, 7] and others, up to the modern ones [3]. A number of monographs are specially devoted to breakdown phenomena [8-10].

Breakdown processes depend on the configuration of the electric field, which, in turn, is determined by the geometry of the gap. The geometry best studied both experimentally and theoretically is the case of flat large-area electrodes, i.e., a uniform field. Here, two types of breakdown are possible, depending on the product of the gas pressure $p$ by the distance $d$ between the electrodes. When this product is small $(p d<$ 200 Torr $\mathrm{cm}$ [3]), the avalanche mechanism proposed by Townsend is applicable. In this case, the electrons moving (drifting) from the cathode to the anode and ionizing the gas produce a series of electron ava- lanches, which create a plasma that fills the discharge gap and transfers the gas into a conducting state. The initial electrons are created at the cathode as a result of ion-electron emission, photoelectric effect, and other processes. This mechanism can be extended to the case of a not completely uniform field, e.g., the field between two coaxial cylinders [8]. At greater values of $p d$ and at sufficiently high gap voltages, the streamer mechanism is realized. Under these conditions, avalanches can form that contain a sufficient $\left(\sim 10^{8}-10^{9}\right)$ number of electrons, the space charge of which distorts the external field, creating regions of high field strength at the ends of the avalanche. In these regions, secondary avalanches are generated due to photoionization by radiation from an avalanche or ionization by fast electrons. This results in the formation of a plasma channel: a streamer, which rapidly (with a speed much higher than the electron drift velocity) grows toward the cathode or anode. The value of $p d$ at which the transition from the Townsend to streamer breakdown occurs depends on the gap voltage. At voltages not much higher than the breakdown voltage, this boundary can be moved up to $p d>2000 \mathrm{~cm}$ Torr [10]. With an increase in the interelectrode distance, when the external field becomes essentially nonuniform, the streamer mechanism can be outperformed by the more favorable (in terms of minimizing the breakdown voltage) leader mechanism. A leader is a conductive channel that grows from the high-voltage electrode to the grounded along the trail left by streamers. The channel is very hot and can cover huge distances (lightning).

Based on the value of $p d$, a breakdown in a long tube at low pressure would have to follow the Townsend or, under strong overvoltage, streamer mechanism. However, the external field in this case is substantially nonuniform: its strength is maximal at the high-voltage electrode (HVE) and drops to zero at the low-voltage (usually grounded) electrode. Consequently, electron avalanches cannot start from the cathode with a positive polarity of the applied voltage and cannot reach the anode with a negative one. Therefore, the avalanche breakdown mechanism is impossible here. This was first pointed out by Seeliger and Bock in 1938 [11]. Following this, in experimental work [12], it was shown that the initial stage of breakdown in a long tube is the passage through it of a localized glow region. In later studies, it was found that this is a region of a high potential gradient, or an ionization wave (IW), which provides the initial conductivity in the gap and the subsequent development of a glowdischarge plasma. Thus, in long tubes under reduced pressure, a special breakdown mechanism associated with the passage of a pre-breakdown ionization wave is realized. Depending on the conditions, its velocity is $10^{5}-10^{7} \mathrm{~cm} / \mathrm{s}$, if the voltage rise rate lies in the range typical of the conditions for the ignition of a glow discharge ( $\sim 1 \mathrm{kV} / \mu$ s and smaller). If the potential of $\mathrm{HVE}$ increases much faster (with a steepness of $\sim 1 \mathrm{kV} / \mathrm{ns}$ or 
higher), then the velocity of the IW can exceed $\sim 10^{9} \mathrm{~cm} / \mathrm{s}$. These are the already mentioned fast IWs.

It should be noted that the above classification of breakdown mechanisms is simplified and corresponds to "pure," limiting situations. In reality, intermediate conditions are possible, when intermediate mechanisms or their combinations are realized. In particular, a streamer breakdown can begin with an avalanche stage [13], ionization waves can be observed in the late phase of the Townsend breakdown [10], streamers can acquire the properties of an ionization wave [3], etc. In particular, if the pressure is reduced at a high voltage rise rate, streamers can gradually transform into fast IWs [14].

In view of the key role played by ionization waves in a breakdown in long tubes, this review begins with a brief description of the discovery and study of these waves.

\section{IONIZATION WAVES}

As noted above, ionization waves are divided into fast and slow [15]. In accordance with this classification, this section is divided into two parts. The breakdown processes discussed in this work are preceded by the propagation of slow waves. However, historically, the first to be detected and then intensively studied were fast IWs (FIWs), which arise in the case of very steep voltage wavefronts. The understanding that the breakdown under typical conditions for glow-discharge ignition is also accompanied by the passage of an IW came much later, as well as their study. Therefore, Section 2 begins with a review of the FIWs.

\subsection{Fast Waves}

The phenomenon, which was later called the ionization wave (IW), was discovered by J.J. Thomson in 1893 [16]. Thomson studied breakdown in a long (very long: of length $15 \mathrm{~m}$, the diameter being of $5 \mathrm{~mm}$ ) glass tube in air at a pressure of 0.5 Torr. The electrodes of the tube were connected to the terminals of an induction coil. It turned out that, under the action of a high voltage, the discharge glow initially does not occur in the entire tube, but only near the high-voltage anode, and then moves to the cathode with a finite velocity. This velocity was measured using a rotating mirror, which reflected radiation from two different points of the discharge and sent it to a measuring telescope. The resulting value exceeded half the speed of light. Almost 40 years later, in 1930, Beams [17] continued these studies. He studied breakdown in a tube $4.9 \mathrm{~m}$ long and $5 \mathrm{~mm}$ in diameter, filled with air or hydrogen at a pressure of $0.05-0.4$ Torr. To obtain a high-voltage (positive or negative) pulse of $20-40 \mathrm{kV}$, a condensed discharge was used. Under the action of a pulse, at the high-voltage electrode, a glow appeared, the front of which first had a conical shape, then, while moving along the tube, became flat and moved with an approx- imately constant velocity. The article gives the values of $(4-5) \times 10^{9} \mathrm{~cm} / \mathrm{s}$ and asserts that the velocity increases with the voltage and does not depend on its polarity. At the moment when the glow front reaches the opposite electrode, a breakdown occurs and a current appears in the discharge circuit. In some cases, after the glow reached the low-voltage electrode, its motion in the opposite direction was observed. The author discusses the possible nature of the observed phenomenon, but does not come to any specific conclusion.

The next, very important step was taken in the works of Snoddy, Beams and Dietrich (1936-1937) $[18,19]$. They were first to study the electrical characteristics of the process in a discharge tube during breakdown with a cathode-ray oscillograph. Its deflecting plates were fed with the potentials of two external ring electrodes. Tubes with a length of $15 \mathrm{~m}$ and an inner diameter of $1.7-18 \mathrm{~mm}$ were filled with air, hydrogen, or carbon dioxide at a pressure of $\approx 0.02-0.2$ Torr. The pulse amplitude was $74-171 \mathrm{kV}$. In [19], optical studies were also carried out. Oscillographic measurements showed that the potential wavefront moves during the breakdown from the high-voltage to grounded electrode, and its velocity coincides with the velocity of the glow front. The range of measured velocities is from $5 \times 10^{8}$ to $\approx 10^{10} \mathrm{~cm} / \mathrm{s}$, depending on conditions. During its motion, the wavefront can slow down or accelerate. The preliminary ionization of the gas increases the velocity several-fold. The wavefront has a finite extent; the electric field strength averaged over this gap reaches $\approx 2000 \mathrm{~V} / \mathrm{cm}$. The potential wave carries a current whose density reaches $4000 \mathrm{~A} / \mathrm{cm}^{2}$. In most conditions, a return wave, moving in the opposite direction with a velocity of $\approx 1 \times 10^{10}$ $\mathrm{cm} / \mathrm{s}$ is detected. When the low-voltage electrode is disconnected from the ground, the return wave disappears, but nothing changes for the primary wave. In the authors' opinion, the wave moves due to the ionization at the wavefront, which requires the presence of electrons preceding it. In the case of a positive wave (with a positive voltage pulse), these electrons can appear as a result of photoionization by radiation from the wavefront or be emitted from the tube wall. A negative wave delivers electrons from the wavefront. These statements are fully consistent with modern concepts. In continuation of these works, Mitchell and Snoddy [20] found that, when the pulse voltage decreases, the wavefront begins to attenuate: its velocity, the current that it carries, and the brightness of the glow decrease. In this work, the discharge tube was placed in a grounded electrostatic shield; such a shield was then used in most studies of the IWs. Among the works of this conditionally early stage, it is also worth mentioning the papers $[21,22]$, in which an IW propagated through a glowdischarge plasma.

Further development of experimental equipment, and above all, diagnostic capabilities, made it possible to achieve significant progress in these studies. Inten- 
sive works in this direction were performed in Moscow (at the Joint Institute for High Temperatures and the Moscow Institute of Physics and Technology (MIPT)), in Arzamas, and Tomsk. Their results are summarized in reviews [15, 23-26]. Currently, these studies are ongoing in France at École Polytechnique (S. Starikovskaya) and in the USA in Columbus (I. Adamovich) and Princeton (A. Starikovskii). In Russia, work in this direction continues at the MIPT (N. Aleksandrov) and in Makhachkala (N. Ashurbekov). These studies are stimulated by the prospects of the practical use of ionization waves in a variety of plasma-chemical technologies, laser physics, for the generation of high-energy electrons, etc. In parallel, theoretical and computational methods for simulating IWs have been developed and improved, which is also reflected in reviews [15, 23-26] and monographs [27, 28 ]. It should be noted that the existing models, as a rule, consider the stage of already formed ionization waves rather than their formation [15]. In addition, it is difficult to describe the mechanism of the appearance of electrons preceding the front of a positive wave; therefore, it mostly common to consider the wave moving in a preionized gas [24].

As already noted, the waves of the type under consideration, having a velocity of $\sim 10^{9} \mathrm{~cm} / \mathrm{s}$, are usually called "fast ionization waves" (FIWs). (In [28], an IW is defined as fast if no appreciable displacement of ions occurs during the characteristic time of its motion.) FIWs arise under a high overvoltage, i.e., when the potential $U$ of the high-voltage electrode is much higher than the minimum value required for breakdown. In this case, the voltage rise rate $d U / d t$ should also be sufficiently large. This condition is on its own necessary for the arising of FIWs. In this case, the initial electric perturbation in a time shorter than the diffusion time creates a large gradient of potential and space charge $[23,29]$. On the other hand, a fast voltage growth allows it to rise to a high level before a breakdown occurs. At such voltages, high-energy (runaway) electrons are generated at the wavefront, which play a significant role in the formation of the wavefront at low pressures [24]. Most commonly, a voltage $U \sim 10-$ $100 \mathrm{kV}$ is used, which can be at least an order of magnitude higher than the breakdown potential. In this case, $d U / d t \sim 1-10 \mathrm{kV} / \mathrm{ns}$ and the width of the leading edge of the pulse is $\tau_{f} \approx 2-5$ ns $[14,30]$. The pulse duration is usually $\tau_{p} \approx 20-50 \mathrm{~ns}$, and the repetition frequency is $f \approx 10-40 \mathrm{~Hz}$. The current carried by the wave has a typical value of $\sim 1 \mathrm{kA}$.

\subsection{Pre-breakdown (Slow) Ionization Waves}

The parameters of a pulse generating a FIW, given in Section 2.1-the amplitude, rise rate, and wavefront duration-are very different from the values usual for the ignition of a low-pressure glow discharge in tubes $\sim 0.1-1 \mathrm{~m}$ long. In this case, the characteristic values of the voltage pulse amplitude are $U_{0} \sim 1 \mathrm{kV}$, i.e., one to two orders of magnitude smaller than those used in the excitation of FIWs. At such voltages, the conditions for generating runaway electrons [24, 31], which play a significant role in the formation of FIWs, are not satisfied. In order to maintain the normal mode of glow discharge, it is necessary to include in the circuit a ballast resistor with $R_{b} \sim 1 \mathrm{k} \Omega$ or higher. Such resistance leads to a delay of the voltage pulse wavefront by $\tau_{f} \approx R_{b} C \sim 1 \mu \mathrm{s}$ ( $C$ is the stray capacitance of the circuit elements). This corresponds to $d U / d t \sim 1 \mathrm{kV} / \mu \mathrm{s}$. Consequently, the steepness of the leading edge of the pulse differs from that typical for FIW excitation by three to four orders of magnitude. Moreover, prebreakdown waves can be excited at $d U / d t$ that are several orders of magnitude lower. An example is given in Fig. 2, where the results of study of a breakdown in a discharge tube $80 \mathrm{~cm}$ long and $1.5 \mathrm{~cm}$ in diameter in neon at a pressure of 0.6 Torr are presented. The breakdown is carried out by a pulse with a linearly growing wavefront $A B$ with $d U / d t=4.7 \mathrm{~V} / \mathrm{ms}$. At the moment of breakdown, at point $B$, a voltage drop at the anode occurred. Section $C D$ corresponds to a steady-state glow discharge, and, at point $D$, the pulse was interrupted. In the lower part of the figure, the optical signals recorded by two photomultipliers from two points of the tube at a distance of $40 \mathrm{~cm}$ from each other are shown. The presence of maxima proves the passage of the IW from the high-voltage anode to the grounded cathode. At the same time, the pulse parameters initiating this wave are very different from those typical of FIW generation. This is especially true for the voltage growth rate and the leading edge duration, which is almost $0.4 \mathrm{~s}$.

Not only in terms of excitation, but also in their properties, such waves are very different from FIWs. For example, the FIW velocity increases with increasing $d U / d t$ [32-34]. In what follows, we will see that, for slow IWs, this is not so. Next, the FIW velocity increases with increasing initial electron density [24, 32]. It will be shown below that the electrons remaining after the previous pulse, on the contrary, can interfere with the generation of the pre-breakdown wave and even block it. It should also be noted that the current carried by a pre-breakdown IW has an order of magnitude of 1-10 mA, i.e., 5-6 orders of magnitude smaller than that in a FIW.

A characteristic feature of slow IWs is a large role played in their propagation by the walls of the discharge tube, in particular, the wall charging process. The models describing these waves $[35,36]$ pay serious attention to the interaction of the plasma with the boundary. At the same time, dielectric walls of the tube do not play a key role in the propagation of FIWs, although can affect them [24]. In particular, an IW can propagate even in the absence of walls [37, 38]. Slow waves can differ from FIWs visually. At a speed of $10^{9} \mathrm{~cm} / \mathrm{s}$, during the lifetime of the excited atoms 

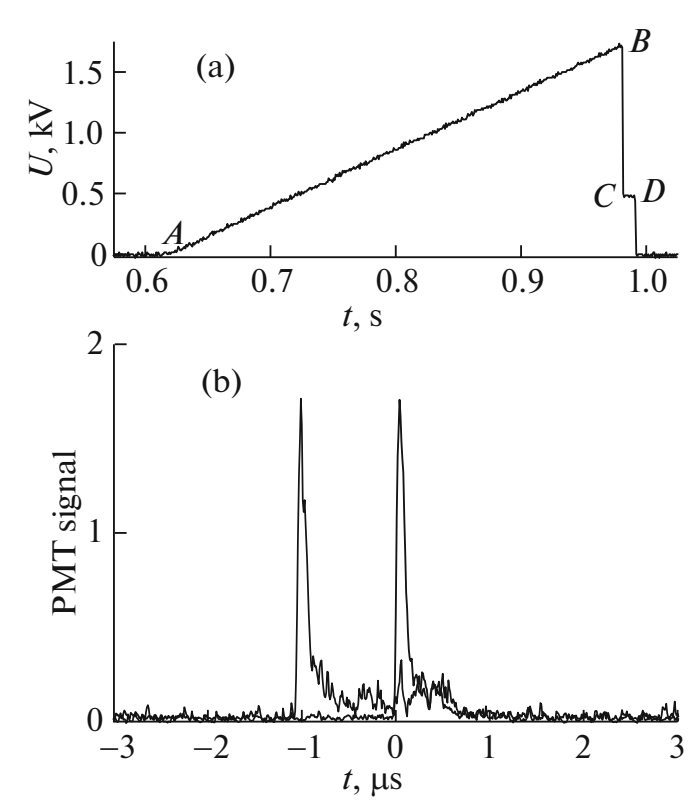

Fig. 2. (a) Time dependence of the anode voltage and (b) emission of an IW. Neon, pressure of 0.6 Torr.

$\left(\sim 10^{-7} \mathrm{~s}\right)$, a wave travels a distance of $\sim 1 \mathrm{~m}$ and, therefore, leaves a trace in the form of a luminous volume (Beams [17] describes it as a cylinder the base of which lies on the HVE). At a speed of $10^{7} \mathrm{~cm} / \mathrm{s}$, this distance is $\sim 1 \mathrm{~cm}$ and the wavefront is detected as a moving peak of the glow (see Fig. 2).

In conclusion, let us briefly dwell on the terminology. In an article [29], Loeb introduced the concept of ionizing waves of potential gradient (before that, they were called potential waves). In subsequent works, the term was reduced to ionizing waves, which fully reflected their physical nature. It remained in this form until the mid-1980s, when the term "ionization waves" appeared in the titles of articles. This replacement is hardly appropriate, because the scope of the second term is much wider. For example, moving striations are also called ionization waves. Nevertheless, this term gradually became prevailing and it is currently used in the literature. Although sometimes, it is impossible to determine what is meant without referring to the context. For example, some articles devoted to ionization waves refer to paper [39], probably because its title contains the words "ionization waves." In fact, it describes the study of moving striations.

\section{STUDIES OF BREAKDOWN IN LONG TUBES AT LOW PRESSURE}

For better understanding, this section is divided into three parts, describing three time periods: (1) until about 1960, (2) 1960-1980s, and (3) 1990s and later. This division reflects to some extent the evolution of research. The first period is the recognition of the problem and the search for the appropriate model; the

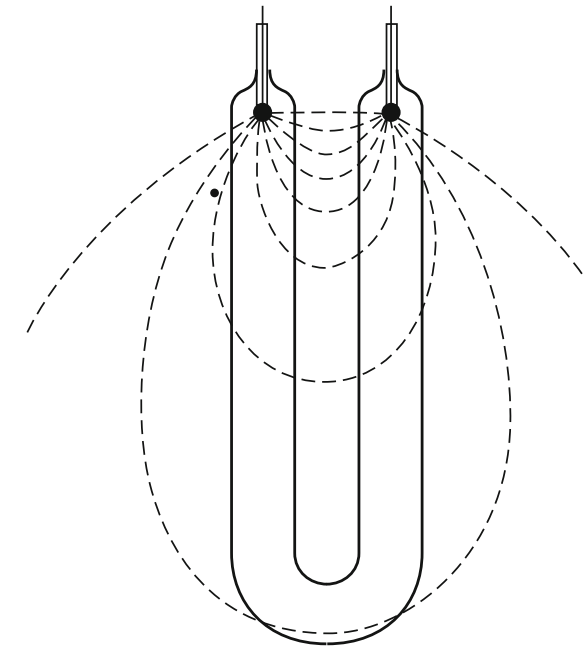

Fig. 3. Electric field lines in a U-shaped tube before breakdown [11].

second is the refinement of the model and the accumulation of experimental data; and the third is research using modern equipment and computing capabilities.

\subsection{Early Works (until 1960)}

The first empirical regularities of breakdown in long tubes were described in 1938 in the book [40]. A year before, in [41], the first theoretical model of such a breakdown, based on the assumption of the uniformity of the electric field in the gap, was proposed. However, in 1938, Seeliger and Bock [11] objected reasonably that, at the time of breakdown, there is no uniform field in a long tube. They escalate the problem, taking a U-shaped tube (Fig. 3), in which the field is concentrated between the electrodes and is absent in the rest of the tube. Therefore, the discharge can be ignited only after the processes forming the longitudinal field and, in their opinion, the ignition process must propagate in the form of a wave. The following year, in 1939, Bartholomeyczeyk [12] conducted a thorough study of the ignition of a discharge in tubes about $50 \mathrm{~cm}$ long and $2-3 \mathrm{~cm}$ in diameter. Helium was mainly studied. One of the electrodes (high voltage) was internal, and the second was in the form of outer ring. Under these conditions, a steadystate discharge was not ignited, but a breakdown occurred. Optical studies of the radiation from the gas in the tube revealed the following picture. Initially, near the HVE, a glowing region resembling a corona discharge appears. Then this region is pushed out and moves in the form of a cloud along the tube until it reaches the opposite electrode. At this moment, a breakdown occurs. The author believes that the cloud during its motion charges the tube wall and thereby creates a guiding longitudinal field.

Regarding the works [11] and [12], one interesting circumstance should be noted. Both of them were 
completed later than the work of Beams et al. [17-19], and even more so after J.J. Thomson [16]. However, in $[11,12]$, there is not a single mention of these studies. It is unlikely that the authors did not know about them. It can be assumed that they considered the conditions for FIW generation to be too far from the usual conditions for discharge ignition.

Then the research on ignition of a discharge in long tubes was developed mainly in the USSR. The main intrigue was the mechanism for creating a guiding longitudinal field. Three versions were considered. In [42-44], it was suggested that such a mechanism is an electron beam. According to the authors, in the initial stage of breakdown, electrons can have a large directed velocity and be focused into a beam by the field created by the space and surface charges. In $[42,44]$, to confirm the existence of such a beam, a magnetic armoured lens was put on a discharge tube at different distances from the cathode and the breakdown voltage $U_{b}$ was measured depending on this distance. Neon at a pressure of $0.1-0.6$ Torr was studied. In [43], for the same purpose, an electrostatic immersion lens was used. The voltage $U_{b}$ turned out to be periodically dependent on the position of the lens $[42,44]$ or on the optical power of the lens [43], which was interpreted by the authors as evidence of the presence of an electron beam focused by the magnetic field. The interpretation of the results of these studies seems doubtful. The formation of an electron beam under the conditions of these experiments is extremely unlikely, at least at a noticeable distance from the cathode. The authors refer to [45], where the presence of such a beam was indicated by $\mathrm{X}$-ray radiation from the anode of the tube. However, it should be noted that the measurements in [45] were performed at a pressure below 1 mTorr (i.e., almost in vacuum) and at a high anode voltage of about $100 \mathrm{kV}$.

Another version of the mechanism for creating a guiding longitudinal field was proposed in [46]. Breakdown in standard fluorescent lamps of various lengths was studied. One of the electrodes was free, and the second was fed with alternating voltage of the industrial frequency and variable amplitude. At a certain value of this amplitude, a glow appeared near this electrode. With a further increase in the amplitude, the glow region first increased and then instantly filled the entire tube. The authors called this state "singleelectrode discharge." In their opinion, this discharge is the first stage of breakdown. It causes ionization and the initial conduction of the gas in the tube. At the same time, it leaves a surface charge on the tube wall. It is this charge that creates the guiding field. In a later article [47], the authors complicated the scheme by combining the concept of a single-electrode discharge with the idea of the dominance of the directional motion of electrons in it over the chaotic one.

The third breakdown mechanism, confirmed by later studies, was proposed by Nedospasov and Novik
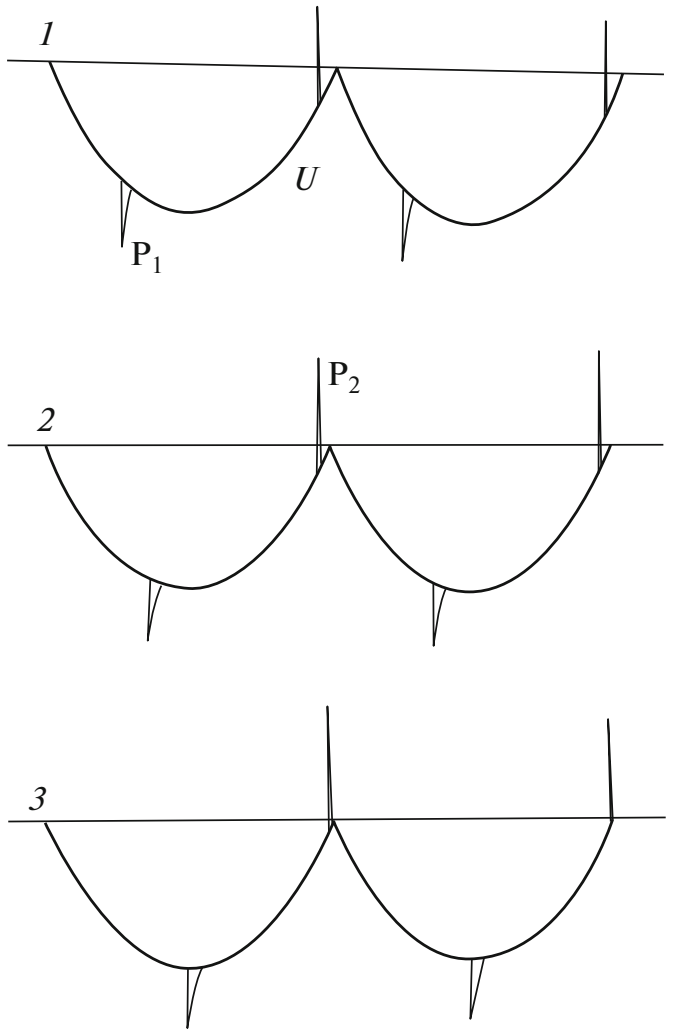

Fig. 4. Oscillograms of current through plates [48]. $P_{1}$ are discharge pulses and $\mathrm{P}_{2}$ are pulses of recharging the tubeplates capacitance; the distance from the HVE is (1) 5, (2) 15 , and (3) $25 \mathrm{~cm}$; $U$ is the HVE potential fed to the plates through the voltage divider.

[48] in 1960. They studied the ignition of a discharge in argon at a pressure of $0.5-10$ Torr in tubes of various lengths and diameters. As in $[12,46]$, one of the electrodes was connected to a voltage source and the other was free. In fact, the role of the second electrode was played by the outer plates placed along the tube at different points. The current through the plates was measured by an oscilloscope. The integral radiation from two points of the tube was detected by two photomultipliers. The HVE was fed either by a sinusoidal or rectified half-wave voltage of variable frequency and amplitude (Fig. 4, $U$ curves). The voltage growth rate was $10^{5}-10^{6} \mathrm{~V} / \mathrm{s}$. The following picture was observed. At a certain voltage, near the HVE, a weak glow arose and current pulses in the circuit of the nearest plate were recorded (Fig. 4, $\mathrm{P}_{1}$ ). With a further increase in voltage, the glow and current pulses appeared at an ever greater distance, the pulses on the farther plates being regularly shifted in time, which evidenced a finite propagation velocity of the process. At a sufficiently high voltage, the process extended to the entire tube. Similar results were obtained for neon. From these oscillograms and from optical measurements, the velocity of the discharge front was found; depending on the conditions, it was $\approx(0.5-5) \times 10^{5} \mathrm{~cm} / \mathrm{s}$. 
The authors draw the following conclusions about the origination of discharge. With increasing voltage, a breakdown occurs between the HVE and the nearby wall section. A current arises, charging the wall to a potential close to the potential of the electrode, and a plasma cloud is formed. Subsequently, the electric field is concentrated mainly between the surface of this cloud and the subsequent sections of the wall. At a sufficient magnitude of this field, the plasma boundary shifts along the tube due to new ionization in the region of a strong field. Thus, the plasma boundary follows the ionization wavefront, carrying an electric field in front of it. In the forming plasma column, weak longitudinal and transverse fields remain, due to which a current flows from the electrode, charging the wall. When ionization extends to the entire tube, the first stage of discharge formation ends. It should be noted that the authors do not use the term "ionization wave," but speak about an "ionization front." In some later works, this term is also not used. They speak about a "pre-breakdown" or the "first" wave. Probably, this emphasizes the fact that this is not about the FIW arising under significantly different conditions.

It should be emphasized that the novelty of this work in the understanding of the breakdown mechanism is the existence of a primary breakdown between the HVE and the wall. It also proposed a computational model in which the development of the discharge is described by a one-dimensional equation of $R C$ line with distributed parameters.

\subsection{Works of the 1960-1980s}

More recent studies have not changed much in understanding the breakdown processes in long tubes. The dependences of the quantitative characteristics of the process on the type of gas and experimental conditions were mainly studied. In almost all works, the velocity of the pre-breakdown IW, $v_{w}$, was measured. The wave is easily detected by radiation from its front. In [49], breakdown was studied in different gases $\left(\mathrm{H}_{2}\right.$, $\mathrm{He}, \mathrm{Ar}$, and $\mathrm{O}_{2}$ ) in a wide range of pressure $p=10^{-2}-$ 100 Torr and in tubes of various diameters and lengths. The dependence $v_{w}(p)$ turned out to be nonmonotonic, with a maximum in the region of $p \sim 1$ Torr. This is similar to FIWs, for which this function is nonmonotonic too [23-25]. On the other hand, a significant difference from FIWs is that the IW velocity proved to be independent of the voltage rise rate in the range $10^{7}-10^{11} \mathrm{~V} / \mathrm{s}$, which is completely unusual for FIWs [32-34].

In $[50,51]$, breakdown processes were studied in a helium-neon laser mixture at $p=4$ Torr in a sitall cell with a diameter of $3.5 \mathrm{~mm}$ and a glass tube with a diameter of $6 \mathrm{~mm}$. The voltage at the high-voltage anode, the cathode current, and radiation from different points along the discharge gap were recorded. A breakdown began with a small drop of the anode volt-

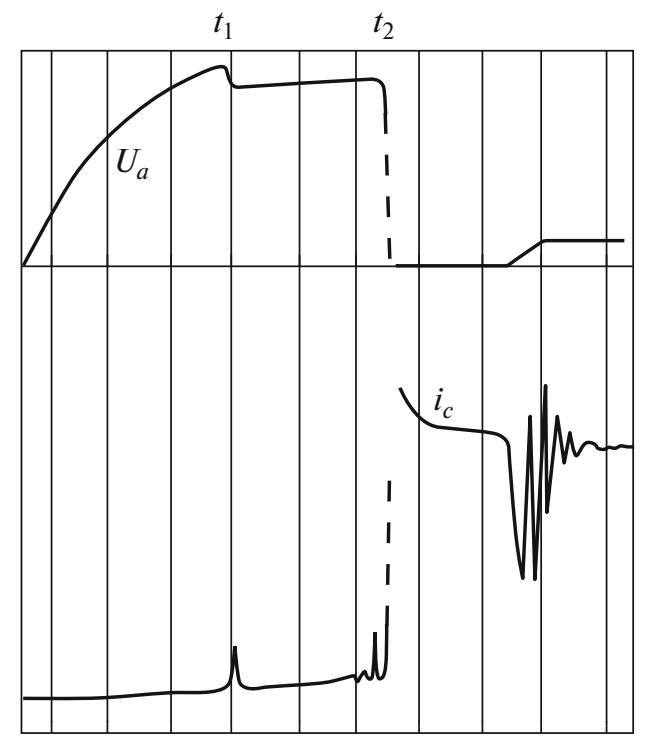

Fig. 5. Oscillograms of the cathode current and anode voltage during breakdown in a $\mathrm{He}-\mathrm{Ne}$ mixture $(p=$ 4 Torr) [51]. Scale: 1 division $=25 \mu$ s.

age $U_{a}$ (Fig. 5) and, simultaneously, with short spikes of the cathode current $i_{c}$ and radiation intensity from the anode region (at a certain time $t_{1}$ ). After some time (at $t_{2}$ ), the same surges of $i_{c}$ and $U_{a}$ occurred, but of a much larger amplitude. The voltage dropped almost to zero, and the current reached a maximum. Then they varied nonmonotonically and eventually reached the level of a steady-state discharge. In the interval between $t_{1}$ and $t_{2}$, the glowing region, i.e., IW, moved from the anode to the cathode. The velocity of the wave was almost constant and, depending on the parameters of the electric circuit, was from $\sim 10^{5}$ to $\sim 10^{6} \mathrm{~cm} / \mathrm{s}$ in the cell and higher than $4 \times 10^{7} \mathrm{~cm} / \mathrm{s}$ in the tube. The shielding of the tube reduced this value by more than an order of magnitude. In [51], the authors propose the following qualitative picture. When moving from the anode, the IW carries in front of it an electric field, in which the electrons produce subsequent ionization and excitation. Electrons preceding the wavefront are born as a result of photoprocesses (photoemission from the tube walls). Behind the leading part of the IW, a conducting plasma column is formed, through which an electron current flows to the anode and the ions charge the distributed plasma-ground (or plasma-screen) capacitance. The current lines close through the bias current. In accordance with this picture, an approximate semi-empirical model is proposed, which, using adjustable parameters, gives correct dependences of the IW velocity on the applied voltage.

The work [52] is the only one in which IWs were registered using Langmuir probes. Two probes with a diameter of $25 \mu \mathrm{m}$ and a length of $3 \mathrm{~mm}$ were located on the tube axis at a distance $l=2 \mathrm{~cm}$ from each other. 
The diameter of the tube is $4 \mathrm{~cm}$, and the studied gases are helium and argon at various pressures and pulse amplitudes. During the passage of an IW, the potential of each probe underwent a jump of $\sim 1 \mu$ s duration. The jumps were shifted relative to each other by the time the wave travels the distance $l$. Hence, the velocity of wave could be found. The range of values obtained was $v_{w} \approx(1-20) \times 10^{6} \mathrm{~cm} / \mathrm{s}$. For both gases, the dependence of the IW velocity $V_{w}$ on pressure was nonmonotonic, with a maximum at $p \approx 2-3$ Torr for $\mathrm{He}$ and $\approx 0.7$ Torr for Ar; the velocity in helium was 24 times lower than in argon. The dependence of $v_{w}$ on the pulse amplitude is approximately linear for helium and essentially non-linear with a power-law shape for argon. The paper proposes an approximate semiempirical model for calculating the IW velocity. For this, the authors use the plasma parameters obtained by processing the probe characteristics, but without an analysis of how applicable the probe theory is for the object under study. The possible influence of the probes and elements of the probe circuit on the IW characteristics is also not discussed. In the subsequent works $[53,54]$, the same authors used the probe method to measure the time dependence of the electron density $n_{e}$ at the initial phase of the discharge in argon at a pressure of $0.3-0.75$ Torr in the same tube. The values obtained reach a steady-state level for 50$70 \mu \mathrm{s}$. In this case, measurements begin 5 or $10 \mu$ s after the voltage is applied. If we use the authors' data for the IW velocity from [52], it turns out that, by this moment, the wave has passed the entire discharge gap. Thus, the data obtained illustrate the ionization multiplication of electrons left by the wave. In both papers, a theoretical model is also proposed.

\subsection{Works of the 1990s and Later}

Since the end of the 1980s, the study of breakdown in long tubes sharply intensified. This was directly related to the start of production and the widespread introduction of compact fluorescent lamps (CFLs), convenient and economical. Most likely, if LED lamps have not appeared, they would completely displace incandescent lamps and become the number one light source. Specifically, the interest in studying the discharge ignition processes in CFLs was connected with the optimization of the operating modes of the lamp itself and the pulsed electronic power circuit. The former, linear lamps were fed with a mains current through a ballast device (usually a choke). Research was conducted in the universities of Eindhoven (Holland) and Augsburg (Germany) and in the laboratories of the leading CFL manufacturers: Phillips and OSRAM. The main results are presented in [35, 36, 55-60], of which [56, 57, 59] are purely experimental, $[35,36,58]$ are computational, and the results of both experiments and modeling are presented in $[55,60]$. The gases under study were the components of the mixtures used in the lamps: argon $[35,56,57,60]$ or argon with mercury vapor $[36,55$, $58,59]$; in [59], there were also neon or krypton additives. The total pressure in all cases was about 3 Torr. The discharge tubes had an internal diameter of $10 \mathrm{~mm}$, the same as of standard CFLs; in [55] a tube with an outer diameter of $32 \mathrm{~mm}$ was used. The cathode or both electrodes of the tube were incandescent. High-voltage pulses are rectangular, with an amplitude $U_{0} \sim 1 \mathrm{kV}$ or smaller and a leading edge duration of $\sim 1 \mu$ s (correspondingly, $d U / d t \sim 1 \mathrm{kV} / \mu \mathrm{s}$ ). In [35, $36,56,57]$, the polarity of the pulses was negative; in other works, pulses of both polarities were studied. The pulse duration in [55] was $10 \mathrm{~ms}$ and, in the rest of the papers, $\sim 100 \mu \mathrm{s}$; the repetition period was $200 \mathrm{~ms}$ (400 $\mathrm{ms}$ in [55] and $10 \mathrm{~s}$ in [59]). With the exception of the latter, in other works, in the intervals between the main pulses, short resetting pulses with an amplitude obviously exceeding the breakdown voltage were applied to the electrodes. Their purpose was to neutralize the surface charge that could remain on the wall if the IW of the previous pulse has not reached the grounded electrode and breakdown has not occurred. In [60], in addition, immediately before the main pulse of positive polarity, a short negative pulse of small amplitude was applied to the anode. It did not lead to a breakdown, but preionized the gas in the anode region and thereby eliminated the statistical delay of the breakdown. With the exception of [59], the discharge tube was surrounded with an electrostatic shield: a grounded metal pipe with a diameter of about $5 \mathrm{~cm}(3.5 \mathrm{~cm}$ in [55]). The purpose of the shield was to eliminate electrical interactions between the discharge and the remaining elements of the equipment, and, in the modeling, impose the boundary conditions for the IW. In addition, the shield made it possible to maintain a constant temperature and humidity inside it. As a rule, two ionization waves were observed: the first (forward) and return. However, the authors of [59] report about only one wave and the authors of [55] about three.

Breakdown IWs were diagnosed by two methods: optical $[56,59,60]$ and electrical: using a capacitive probe $[55,57,60]$. In the first case, an IW was diagnosed by the radiation from its wavefront, and, in the second case, by the potential of the wall on which the wave left a charge. In [59], the radiation of the wave was recorded using 8 photomultipliers placed along its path, and, in $[56,60]$, using an intensified CCD camera. The capacitive probe in [55] was an outer ring mounted in an electrostatic screen and connected to it through a low ohmic measuring resistor. Such a scheme minimized the disturbance introduced by the probe, but had a low spatial resolution due to the gap between the probe and the tube. In [57,60], such a gap was absent and the perturbation introduced in this case was minimized by the special design of the load resistor and the use of an electronic feedback circuit. 
Both methods made it possible to measure the IW velocity $[55,56,59,60]$. The range of values obtained is $V_{w} \sim 10^{5}-10^{7} \mathrm{~cm} / \mathrm{s}$. In all cases, the velocity increased with increasing pulse amplitude $U_{0}$. In [55], it varies as $U_{0}^{q}$, where $q \approx 6$ for positive polarity and $q \approx 1.5$ for negative polarity, as a result of which, for $U_{0}<500 \mathrm{~V}$, the negative wave is faster than the positive wave and, for $U_{0}>500 \mathrm{~V}$, vice versa. In [60], the dependence $v_{w}\left(U_{0}\right)$ is close to linear and, for a positive wave, it is also steeper than that for a negative one; therefore, these lines intersect at $U_{0} \approx 550 \mathrm{~V}$ (Fig. 6). In [59], for $U_{0}=800-1100 \mathrm{~V}$, the positive wave is faster than the negative wave. As the wave moves, its velocity decreases: the lower $U_{0}$, the faster the decrease. At a sufficiently low $U_{0}$, it may occur that the wave does not reach the grounded electrode and disappears at an intermediate point $[56,60]$. In this case, a breakdown of the entire tube and a discharge ignition do not occur. Here, a "memory effect" arises [60], which affects the breakdown processes; in particular, the next breakdown may not occur at all. The authors relate this effect to the surface charge left by the wave. To neutralize this charge, in the interval between the main pulses, additional short pulses with an amplitude large enough to ignite a discharge, which will remove the wall charge, were supplied to the electrodes. Based on these observations, the following statement is formulated: a necessary condition for a complete breakdown of the tube and a discharge ignition is that the pulse be long enough so that the IW can pass the entire gap during the pulse.

The main purpose of using a capacitive probe was to obtain information about the electrical characteristics of the IW. In [55], the bias current to the probe was measured. The integration of the current over time gave a charge, and, by dividing the charge by the capacity of the tube-screen system, the potential of the wall was obtained. Measurements showed that both quantities, after the start of the pulse, reach steady-state values at a distance from the HVE on the order of the tube diameter, and the potential reaches the amplitude value of the pulse voltage. After reaching the grounded electrode, they decrease (in absolute value), which the authors relate to the return wave moving toward the HVE and partially discharging the wall. At the time of its arrival at the HVE, the third wave is generated, propagating in the original direction. In [60], the probe touched the tube surface and, thanks to the corresponding electronic circuit, made it possible to directly measure the wall potential. Differentiation with respect to the axial coordinate gave the longitudinal electric field strength $E$. The curves presented in [60] give the time dependence of $E$. The curves exhibit oscillations, the cause of which is not discussed. In a negative wave, $E=100-115 \mathrm{~V} / \mathrm{cm}$ for the range $U_{0}=400-800 \mathrm{~V}$. For a positive wave, $E=$ $150-200 \mathrm{~V} / \mathrm{cm}$ for $U_{0}=500-700 \mathrm{~V}$, except for the

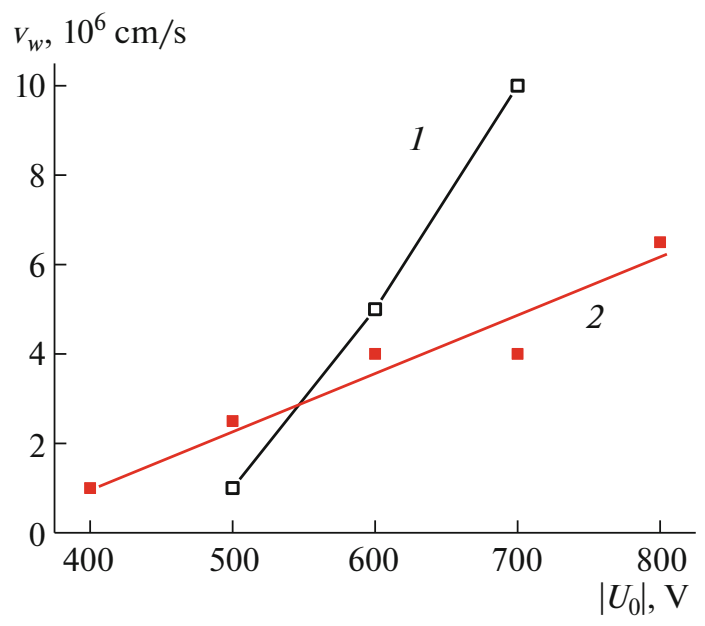

Fig. 6. IW velocity vs. pulse amplitude for the (1) positive and (2) negative polarity (according to [60]). Argon, $p=$ 3 Torr.

moment when the wave arrives at the cathode at $U_{0}=$ $500 \mathrm{~V}$; at this point, $E=283 \mathrm{~V} / \mathrm{cm}$. Data on the bias current and wall charge were also obtained.

In [59] and [60], it was found that the breakdown potential of $U_{b}$ depends on the pulse polarity, but the specific data of these studies differ significantly. In [59] ( $\mathrm{Ar}-\mathrm{Ne}$ and $\mathrm{Ar}-\mathrm{Kr}$ mixtures), $U_{b}$ is higher for negative polarity. Moreover, it is said that, in this case, it is almost never possible to realize a breakdown. In [60] (Ar), the situation is opposite: for positive and negative voltages, $U_{b}=+455$ and $-300 \mathrm{~V}$, respectively.

As mentioned above, in a number of studies, model calculations of various stages of breakdown are carried out. The calculations performed in [55] are based entirely on the Nedospasov and Novik model [48]. The discharge gap is simulated by a set of 125 seriesconnected $R C$ circuits, where $R$ describes the resistance of the plasma column section, and $C$ describes its capacity relative to the ground. The difference from the model [48] consists only in the fact that $R$ is assumed to be time-dependent. Volume ionization processes are described by a very primitive semiempirical model. Nevertheless, it implies the very fact of the feasibility of a self-sustaining motion of the plasma boundary, i.e., the wavefront. However, it should be emphasized that only a negative wave is considered and it is assumed that charged particles are initially present in the gas. In [35] (breakdown in argon), the reactions in the volume are considered more correctly. The hydrodynamic approximation is used, continuity equations for charged particles are written with allowance for various ionization processes, including those involving excited atoms, Poisson equation taking into account the presence of electrodes and a dielectric wall, and processes at the plasma-wall interface. The rate constants of the processes are considered as a function of the mean energy, which is 
related to the parameter $E / p$ using the calculated electron energy distribution function. The result of the calculations is a set of axial dependences of electrical parameters (surface charge, field strength, potential, electron density, and mean electron energy), the velocity of the ionization wavefront at different times, as well as the dependence of the calculated quantities on the parameters: pulse amplitude, pressure, tube diameter, etc. Only a negative wave is considered, and the presence of a heated cathode, emitting initial electrons, is assumed. The results qualitatively agree with the experimental picture of the IW motion. In [36], similar calculations were performed for a mixture containing argon with mercury vapor. It turned out that the Penning ionization reaction of mercury atoms can significantly increase the electron density and affect the velocity of the wave, especially the return one. In [58], a similar approach is used for the case of periodically repeated pulses of alternating polarity of a rather high frequency, up to $120 \mathrm{kHz}$, i.e., for the region typical of CFL power supply. Symmetrical trapezoidal pulses are considered. In this case, the IWs propagate alternately in opposite directions, and the picture is complicated by the fact that the positive and negative waves have different velocities. The process of establishing the lamp parameters and their dependence on the repetition rate and pulse amplitude is theoretically studied. When analyzing the ionization in the wave, the authors neglect stepwise processes, which, at a high pulse repetition rate, may be incorrect.

A different approach to modeling the IW motion is proposed in [60]. The wavefront is approximated by a rectangular region $F$ with a high electric field strength. In front of it, the field is zero, and after it (in the plasma wake), finite but small. Inside $F$, avalanche (Townsend) ionization occurs. Electrons disappear on the tube wall as a result of free diffusion. In the case of a negative wave, the ionization rate is equal to the electron loss rate. The motion of the IW is possible due to the electron drift flow directed from the front to the anode. The electron density in the conducting wake of the wave is maintained due to the arrival of electrons from the cathode. The equality of the ionization and diffusion rates determines the field strength at the wavefront, and the velocity of the wave is determined by the rate of charging the tube capacitance relative to the ground (as in [48]). In the case of a positive wave, seed electrons cannot be caused by the electron drift from the wavefront. Analyzing their possible sources, the authors stop on photoelectron emission from the tube walls under the action of UV resonant radiation of the wave. Since the quantum yield of the photoelectric effect is most likely small, a high rate of excitation of resonant levels is needed; this requires a higher electric field strength at the wavefront than in the case of a negative wave, which is consistent with reality. It should be noted that, evaluating the electron photoemission efficiency, the authors neglect the reabsorption of photons. Nevertheless, the model using fitting parameters gives correct values of the IW velocity and its time dependence.

In the relatively recent experimental works [61, 62], the stages after the passage of the ionization wave-the ignition of a glow discharge with the subsequent transition to the arc mode-were investigated. A U-shaped fluorescent lamp $1 \mathrm{~m}$ long and $17 \mathrm{~mm}$ in diameter was studied. At a distance of $1 \mathrm{~cm}$ from the lamp, there was a grounded metal plate, the presence of which facilitated ignition. The lamp was powered by a sinusoidal voltage with a frequency of $25 \mathrm{kHz}$ and an effective voltage of 400-600 V. The so-called cold start was studied, when the cathode of the lamp is not heated by an external current as in the traditional scheme. This mode is more economic and eliminates the pause necessary for heating the cathode. The time course of the voltage and discharge current after turning on the voltage was recorded. From the oscillograms obtained, one can trace the entire evolution of the discharge ignition: the IW propagation interval, ignition of a normal glow discharge, its transition to the anomalous mode accompanied by heating the cathode by the discharge current, and, as a result, the ignition of an arc. The dependences of the energy deposition to the discharge and the lifetime of the discharge in glow form on the supply voltage are obtained.

\section{MANIFESTATION OF THE WAVE NATURE OF BREAKDOWN DURING DISCHARGE IGNITION IN LONG TUBES}

The fact that the initial stage of breakdown in long tubes is the passage of an ionization wave through the discharge gap leads to a number of features differing it from the "waveless" Townsend breakdown. One of them has already been mentioned: this is the dependence of the breakdown potential on the polarity of the applied voltage $[55,59,60]$, in other words, on which of the electrodes-the cathode or the anode-is grounded or at least is at a low potential relative to the ground.

The need for grounding is caused by the specifics of breakdown in long tubes: the presence of primary breakdown on the wall as the initial stage of discharge ignition. This circumstance is illustrated by Fig. 7. In it, $r_{1}$ and $r_{2}$ are the limiting (ballast) resistors (one of them is usually absent, i.e., its resistance equal to zero) and $R_{1}$ and $R_{2}$ are the resistances of the insulation (leakage) of the terminals of the power source. Usually, $R_{1} \sim R_{2} \gg r_{1}, r_{2}$. Let the potential of the ground and the surrounding space be zero. Before the voltage is turned on, the potentials of both electrodes and the tube wall are also zero. Suppose that none of the electrodes is grounded. Then, after turning on the voltage $U$, but before the current appears, the potentials of the electrodes will be $\left[+R_{1} /\left(R_{1}+R_{2}\right)\right] U$ and $\left[-R_{2} /\left(R_{1}+\right.\right.$ $R_{2}$ )] $U$, while the potential of the wall is still zero. Due to the uncertainty and instability of the resistances 


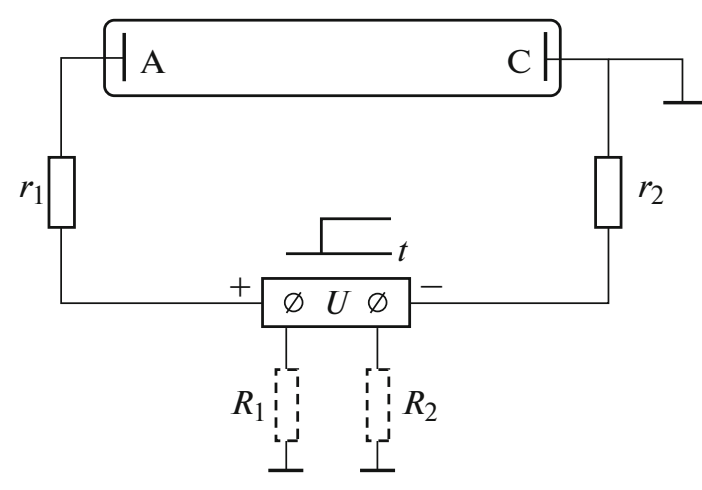

Fig. 7. Schematics of connecting a discharge tube at a positive pulse polarity.

$R_{1}$ and $R_{2}$, the potential of the electrodes relative to the wall will also be undefined, which will lead to unpredictability and irreproducibility of the primary breakdown. This will not happen if one of the electrodes of the tube is grounded, directly or through a low resistance. The polarity of the ungrounded electrode determines the direction of propagation and the properties of the IW and, ultimately, the breakdown characteristics. This is obviously not the case of the Townsend breakdown, in which, if the discharge gap is symmetrical, only the potential difference between the electrodes is important, and the polarity of the grounded electrode, as well as the presence of grounding, do not play a role.

In addition to the dependence of the breakdown potential on the sign of voltage, there are other specificities of the breakdown processes in long tubes, caused by its wave mechanism. They are considered below.

\subsection{Electrical Signals in the Discharge Circuit}

The time dependence of the electrical characteristics of the discharge (currents and voltages) can carry useful information about the breakdown process. Despite this, such studies are few. In [51], oscillograms of the voltage at the high-voltage anode and of the current through the cathode during breakdown in a tube filled with a $\mathrm{He}-\mathrm{Ne}$ mixture are shown (Fig. 5), and, in [55], the time course of the current through the high voltage cathode for the $\mathrm{Ar}-\mathrm{Hg}$ mixture is demonstrated. More detailed and illustrative results were obtained in $[63,64]$. Figure 8 shows oscillograms recorded in the study of breakdown in a tube $80 \mathrm{~cm}$ long and $23 \mathrm{~mm}$ in the inner diameter in argon with mercury admixture at a total pressure of 3 Torr and a $\mathrm{Hg}$ vapor pressure of $1 \mathrm{mTorr}$ with a grounded cathode and a ballast resistance of $20 \mathrm{k} \Omega$. At the time $t_{0}$, a pulse with an amplitude $U_{0}=2 \mathrm{kV}$ is fed to the anode. At the same time, a surge in the anode current, caused by charging the tube-ground capacitance, is seen. At the point $t_{1}$, the anode current sharply increases: a primary breakdown occurs. Simultaneously, the anode voltage

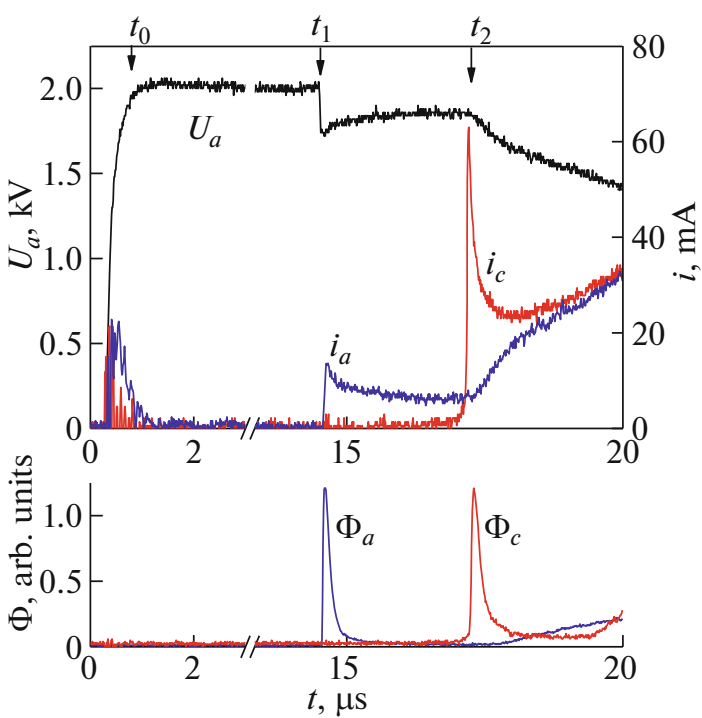

Fig. 8. Breakdown in an $\mathrm{Ar}-\mathrm{Hg}$ mixture $(p=3$ Torr and $\left.p_{\mathrm{Hg}}=1 \mathrm{mTorr}\right)$. Oscillograms of the anode voltage, the anode $\left(i_{a}\right)$ and cathode $\left(i_{c}\right)$ currents, and the integral emission intensity near the anode $\left(\Phi_{a}\right)$ and cathode $\left(\Phi_{c}\right)[63]$.

abruptly decreases by the value of the drop in the ballast resistance. For $2.5 \mu \mathrm{s}$, a current flows through the anode; since, in this case, the cathode current is zero, the anode current charges the wall, and its circuit is closed through the plasma-ground capacitance, i.e., through the bias current. At the time $t_{2}$, a cathode current appears and the breakdown is completed. The anode and cathode currents increase, approach each other, and reach a steady-state level. In the interval from $t_{1}$ and $t_{2}$, the luminescence peak moves from the anode to the cathode; i.e., the IW generated at the anode at the time of primary breakdown moves and arrives at the cathode at the moment of complete breakdown. The time interval from $t_{0}$ to $t_{1}$ is the time delay of the primary breakdown. From pulse to pulse, it changes randomly, as is also observed during the breakdown of short gaps [8].

Similar curves for a pulse of negative polarity are shown in Fig. 9. The most noticeable difference from the previous picture is the absence of a peak in the signal of the current in the low-voltage electrode (in the given case, anode) at the time of complete breakdown.

The data presented in these figures were obtained in a tube with a cold (non-incandescent) high-voltage electrode. With a positive pulse polarity, the low-voltage cathode was incandescent, but this did not affect the shape of the curves. Incandescence of the cathode prevented its sputtering and destruction due to ion bombardment. Figure 10 demonstrates the effect of the heating of the high-voltage cathode. In this case, the primary breakdown occurs at the leading edge of the pulse, i.e., there is no delay of breakdown. This is quite expectable, since the time delay (more precisely, 


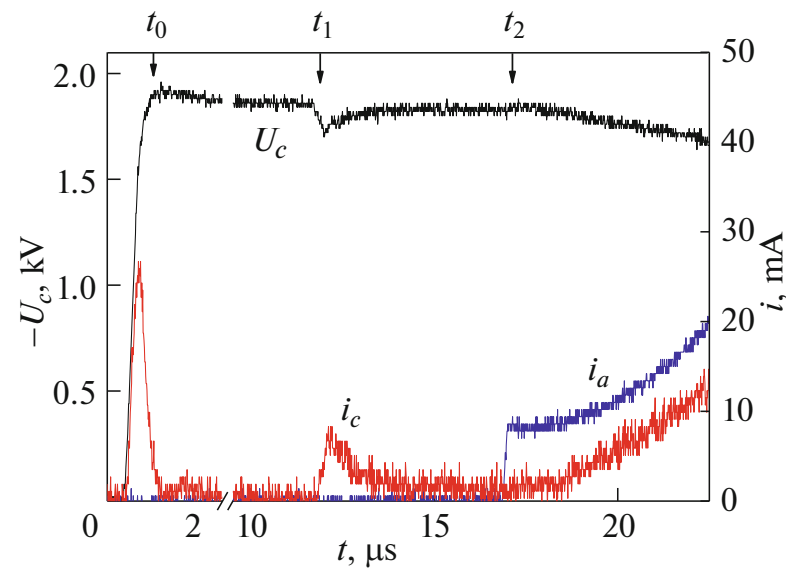

Fig. 9. Breakdown in an $\mathrm{Ar}-\mathrm{Hg}$ mixture by a pulse of negative polarity. Oscillograms of cathode voltage and the cathode $\left(i_{c}\right)$ and anode $\left(i_{a}\right)$ currents [64]. The conditions are the same as in Fig. 8.

its statistical component: under the given conditions, the main one $[65,66])$ is determined by the frequency of appearance of the electrons initiating the breakdown. When the cathode is heated, this frequency becomes very large. The delay of the leading edge of the voltage pulse in Fig. 10 is caused by the capacity of the heating circuits.

\subsection{The Effect of Shielding of the Tube on the Breakdown}

As noted above, in many studies of breakdown, the discharge tube was surrounded with a conductive grounded shield. The purpose of the shield was to eliminate the electrical effect of other elements of the equipment on the discharge. Although this effect is indeed eliminated, it is replaced with an effect of the shield. To determine the degree of this effect, experiments with conducting shields were carried out in [63-65]. The shields had the form of cylinders of various diameters: from 3 to $67 \mathrm{~cm}$ and a length equal to that of the discharge tube $(80 \mathrm{~cm})$. Their ends were open; therefore, the shielding of the tube was not complete. The shields were grounded either directly or, for the purpose of measuring the current in the shieldground circuit, through a low measuring resistance. It can be seen in Fig. 11 that even incomplete shielding significantly affects the breakdown. The presence of the shield dramatically enhances the dip of the anode voltage during the initial breakdown and increases the anode current during the propagation of the ionization wave. The spike of the cathode current, on the contrary, decreases, and can even disappear completely (Fig. 12). The smaller the diameter of the shield, the stronger these effects. For a shield of maximum diameter $(67 \mathrm{~cm})$, they are almost invisible.

Obviously, the effect of the shield is due to an increase in the capacitance $C_{S}$ between the tube and

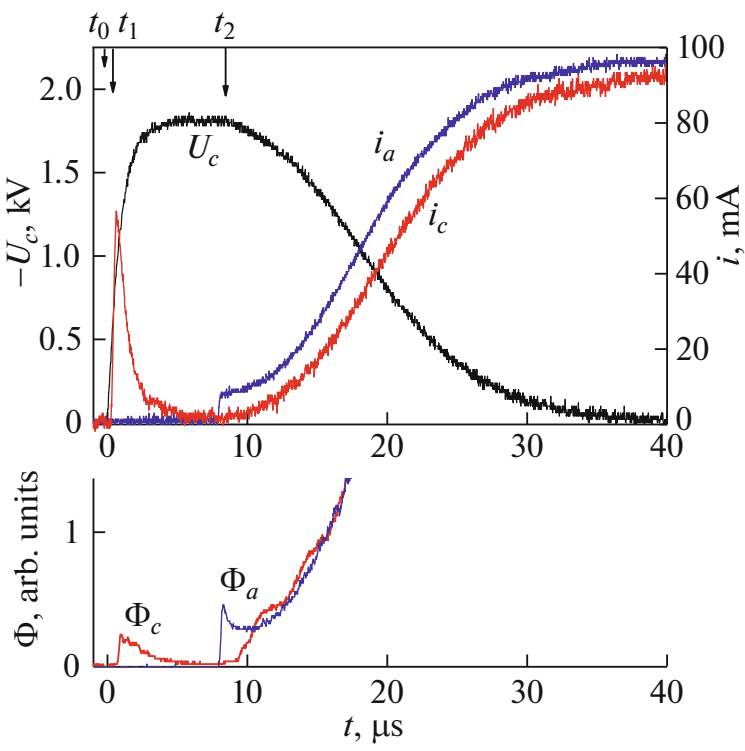

Fig. 10. Breakdown in an $\mathrm{Ar}-\mathrm{Hg}$ mixture by a pulse of negative polarity with cathode heating. Oscillograms of cathode voltage, cathode $\left(i_{c}\right)$ and anode $\left(i_{a}\right)$ currents, and emission intensity near the anode $\left(\Phi_{a}\right)$ and cathode $\left(\Phi_{c}\right)$

[64]. The conditions are the same as in Fig. 8.

the ground. Through this capacitance, the bias current flows during the initial breakdown and the motion of the ionization wave. The presence of a shield increases this capacitance and decreases its impedance, which leads to an increase in the anode current and a voltage jump at the time of the initial breakdown. At the final stage of breakdown, this capacitance, being charged, counteracts the completion of the breakdown: it reduces the jump of the cathode current or smooths it. Figure 12 also shows the waveform of the current $i_{s}$ in the shield-ground section. In fact, in this way, the bias current going to ground is recorded. In the interval from the initial breakdown to the appearance of the cathode current, the shield current is equal to the anode current. After the appearance of the cathode current, as expected, $i_{s}=i_{a}-i_{c}$.

Figure 13 illustrates the effect of shielding on the mean velocity of the ionization wave. This effect depends on the size of the shield. With a sufficiently large diameter $d_{s}$, the effect disappears. With smaller $d_{s}$, the dependence $v_{a v}\left(d_{s}\right)$ is nonmonotonic. In [63], the following qualitative explanation was given. On the one hand, a decrease in $d_{s}$, i.e., an increase in the capacitance $C_{S}$, leads to an increase in the energy deposited in the primary discharge, which can lead to an increase in the initial velocity of the wave. On the other hand, the wave during its motion must charge this capacitance; therefore, an increase in $C_{S}$ must slow down the wave.

Thus, the shielding of the tube, excluding the electrostatic interaction of the discharge with external 


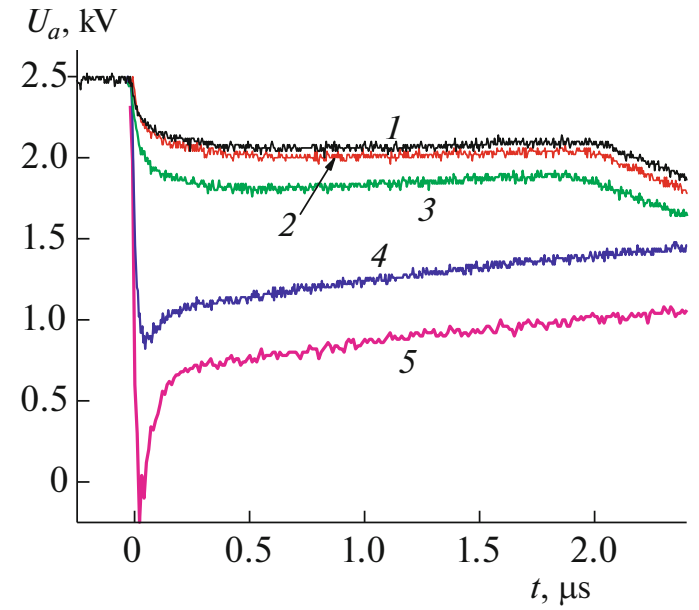

Fig. 11. Time dependences of the anode voltage during breakdown in an $\mathrm{Ar}-\mathrm{Hg}$ mixture in a tube (1) without a shield and with a shield with a diameter of (2) 67 , (3) 25 , (4) 5, and (5) $3.3 \mathrm{~cm} \mathrm{[65].} \mathrm{The} \mathrm{conditions} \mathrm{are} \mathrm{the} \mathrm{same} \mathrm{as}$ in Fig. 8. (C) IOP Publishing. Reproduced with permission. All rights reserved.

devices, leads to a much stronger interaction with the shield. The shields described here did not provide full shielding; it is clear that, otherwise, its effect is even stronger. This means that the results of studying a breakdown in a shielded tube depend not only on the characteristics of the discharge gap, the type of gas, etc., but also on the geometry of the shield. In particular, these results may not be applicable for an unshielded tube. By the way, it is precisely such tubes that are used in compact fluorescent lamps.

\subsection{Breakdown Voltage}

The breakdown voltage or breakdown potential $U_{b}$ is the most important characteristic of the discharge gap. In the Townsend breakdown in a uniform field, the breakdown potential is found from the Townsend criterion and its value for a given gas is determined by the similarity parameter $p d$ (Paschen curves) [3, 8]. For a cylindrical discharge tube of length $l$ and radius $r$, it seems that another similarity parameter, $r / l$, appears. If we assume that the field in such a tube is still uniform, then the only new factor will be the escape of electrons from the gap in their radial diffusion and the role of this process can be determined by the parameter $r / l$. In the works of Lisovskiy et al. [67, 68], a modified Paschen law was proposed, which takes into account the radial diffusion of electrons and, according to which, $U_{b}$ is determined by the parameters $p l$ and $l / r$. It can be seen from Fig. 14 that $l / r$ is indeed a similarity parameter. However, it should be borne in mind that, in this example, the distance between the electrodes is almost equal to their diameter: $l / 2 r=1.2$; i.e., this is a case of a weak nonuniformity of the field. On the whole, the approach of [67,

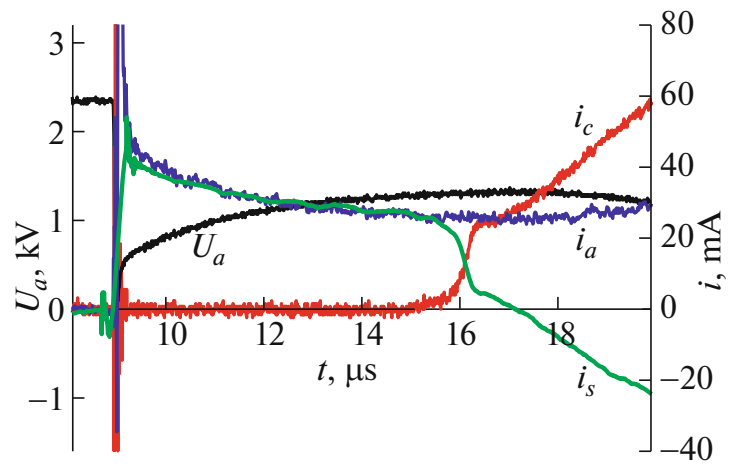

Fig. 12. Time dependences of the anode voltage and the anode $\left(i_{a}\right)$, cathode $\left(i_{c}\right)$, and the shield $\left(i_{s}\right)$ currents. The diameter of the shield is $3.3 \mathrm{~cm}$. Ar- $\mathrm{Hg}$ mixture [63]. The conditions are the same as in Fig. 8.

68] assumes the field uniformity and, therefore, is not applicable to long tubes, where the field is essentially nonuniform and the wave mechanism of breakdown is realized.

In a long tube, the process begins with a primary breakdown between the HVE and the nearest section of the tube wall [48]. Therefore, the first breakdown condition is as follows: the amplitude $U_{0}$ of the pulse applied to the HVE should be large enough for the primary breakdown to occur and the pre-breakdown IW to start. Further on, it is necessary that the IW pass the entire gap and reach the low-voltage electrode. If $U_{0}$ is not high enough, it may turn out that the wave does not reach the grounded electrode and disappears at an intermediate point $[56,60]$. In this case, the breakdown of the entire tube will not occur. This is the second condition for $U_{0}$. In addition, this imposes a con-

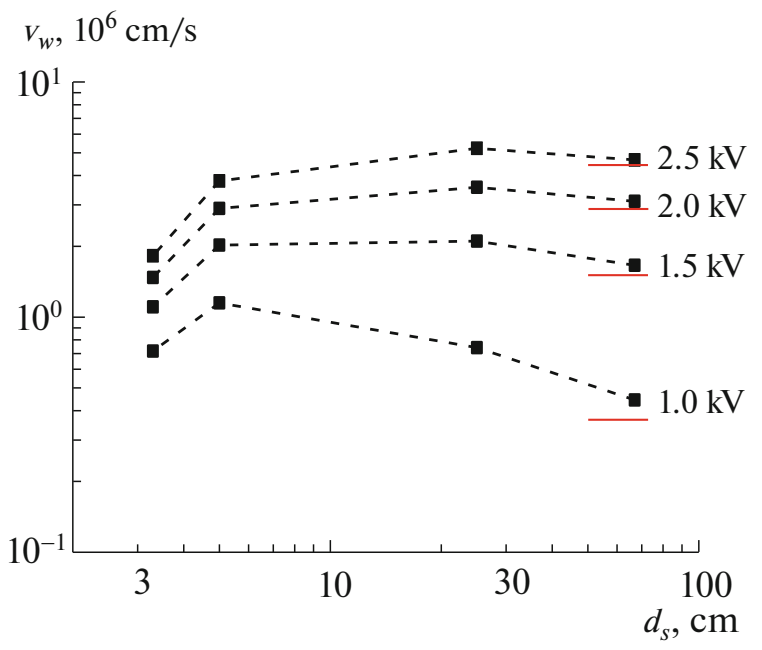

Fig. 13. Length-averaged IW velocity vs. the diameter of the shield at different voltage pulse amplitudes; horizontal lines: represent measurements without a shield. $\mathrm{Ar}-\mathrm{Hg}$ mixture, positive pulse [63]. The conditions are the same as in Fig. 8. 


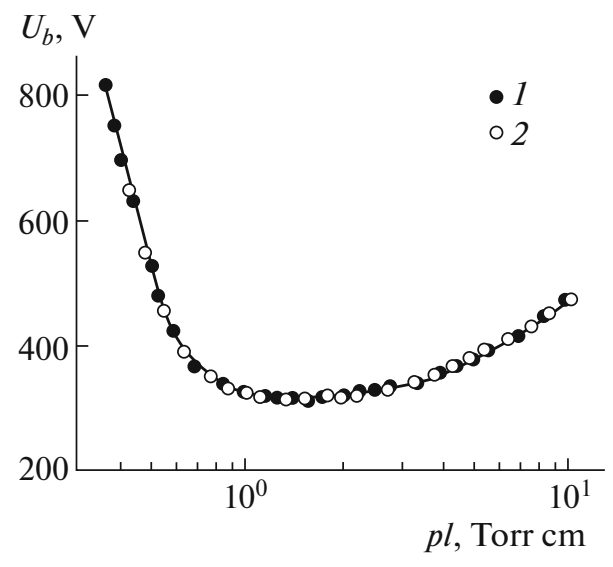

Fig. 14. Modified Paschen curve for argon at $l / r=2.4$ [67]; $l=(1) 1.1$ and (2) $3.3 \mathrm{~cm}$.

dition on the voltage pulse duration [60]. If, along with a breakdown, the ignition of a steady-state discharge is required, then $U_{0}$ should be sufficient to maintain it. The first condition obviously depends on the geometry of the region near the HVE (tube diameter, electrode size, etc.) and does not depend on the length of the discharge gap. The second condition is determined by all these parameters, including the length. For the third condition, cathode material, tube length and diameter, etc. are important. These three conditions are practically independent, and, therefore it is impossible to establish a general regularity such as Paschen law, which would connect $U_{b}$ with the parameters of the discharge gap.

The wave, due to the "concentration" of the electric field at its front, is an effective mechanism for creating the initial ionization in the gap. In this, it is similar to moving striations in the positive column of a discharge, where the field is concentrated in the head of the striation, due to which the voltage drop across the column is lower than if the field were uniform [3]. In the same way, an IW can reduce the breakdown potential. This is illustrated by the following experiment [46] (Fig. 15). Using two plates connected to the electrodes of the tube, the field in it is made close to uniform. In this case, the breakdown potential increases: the longer the gap, i.e., the greater the nonuniformity in the tube without plates, the larger the increase: from $20 \%$ for $l=20 \mathrm{~cm}$ to 2.2 times for $85 \mathrm{~cm}$.

Usually, the breakdown voltage is understood as the so-called static breakdown voltage $U_{S}$. This is the minimum voltage at which a self-sustained form of discharge is maintained [69]. The classical Townsend breakdown condition for short gaps refers to this quantity. If, starting from zero, the interelectrode voltage $U$ is continuously increased with a rate $d U / d t$, then the value of $U_{b}$ at which the breakdown will occur, generally speaking, will exceed $U_{S}$. This is due to the delay of the breakdown relative to the moment when $U=U_{S}$, as a result of which

$$
U_{b}=U_{S}+(d U / d t) t_{d},
$$

where $t_{d}$ is the breakdown time delay. If we measure $U_{b}$ at various voltage rise rates and then extrapolate the dependence obtained to the zero of $d U / d t$, we obtain the value of $U_{S}$ [69]. The time delay of the breakdown can be represented [8] as the sum of two quantities: statistical time delay $t_{s}$ and formative time $t_{f}$ :

$$
t_{d}=t_{s}+t_{f} \text {. }
$$

The statistical delay is the time during which, in the gap, an electron appears that initiates an avalanche, the development of which leads to a breakdown (effective electron [69]); therefore

$$
t_{s}=z^{-1}
$$

where $z$ is the frequency of appearance of effective electrons. The quantity $t_{s}$ is stochastic and can fluctuate in a wide range. The formative time $t_{f}$ is the interval from the moment of the appearance of such an electron to the current jump in the discharge circuit, i.e., to the breakdown. The stochasticity of $t_{s}$ leads to fluctuations in the total time delay $t_{d}$. In turn, the scatter of $t_{d}$, according to relationship (1), leads to a scatter of the values of the breakdown voltage $U_{b}$, for which, in this case, the term "dynamic breakdown voltage" is introduced (sometimes this term is used for the quantity obtained by averaging over a large number of breakdowns).

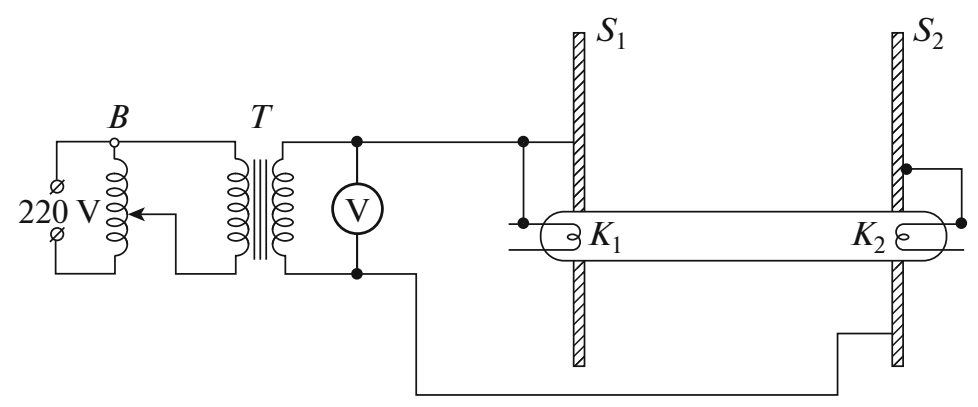

Fig. 15. Schematics for studying breakdown in a field close to uniform [46]; $S_{1}$ and $S_{2}$ are metal plates. 
It is believed that the primary breakdown in a long tube between the HVE and the nearest section of the tube wall proceeds according to the Townsend mechanism [60]; therefore, relationships (1) and (2) hold also for it. Since the moment of breakdown of the entire gap is separated from the initial breakdown by the time of passage of the ionization wave (or waves), the total time delay increases by this value:

$$
t_{d}=t_{s}+t_{f}+t_{w}
$$

where $t_{s}$ and $t_{f}$ refer to the primary breakdown and $t_{w}$ is the time of the IW motion. As in the case of a short discharge gap, the scatter of $t_{s}$ leads, according to relationship (1), to a scatter of the breakdown voltage $U_{b}$. This, in turn, causes a scatter of the pre-breakdown IW velocity $v_{w}$. Experiment shows that the correlation between $U_{b}$ and $v_{w}$ is preserved. Figure 16 shows the dependences of the IW velocity on the breakdown voltage at various values of $d U / d t$ in nitrogen (6 values in the range $0.5-7.0 \mathrm{kV} / \mathrm{ms}$ ) and helium (10 values in the range $3-140 \mathrm{kV} / \mathrm{ms}$ ). Points of different configurations correspond to different values of $d U / d t$, and the range for points with a given $d U / d t$ is due to the scatter of $U_{b}$ and $v_{w}$. It can be seen that all points fit into a common curve, regardless of $d U / d t$. Hence, $v_{w}$ does not depend explicitly on $d U / d t$, although it depends on $U_{b}$. For fast IWs and, accordingly, high voltage rise rates $(d U / d t>0.1 \mathrm{kV} / \mathrm{ns})$, this is not so: the velocity of the wave increases with increasing $d U / d t$ [32-34].

The time $t_{s}$ and, therefore, the scatter of $t_{d}$ and $U_{b}$ can be suppressed using an additional source of electrons initiating the primary breakdown. With a negative potential at the HVE, this can be thermal emission from a heated cathode [55-57,60]. The same result is obtained from irradiation of gas or the surface of the high-voltage cathode with UV, X-ray, or gamma radiation [8]. With a positive potential of the HVE, this effect can be achieved by illuminating the anode region of the tube with radiation with a wavelength shorter than $\approx 500 \mathrm{~nm}[65,66,71,72]$. This issue will be discussed in detail below.

As follows from (1), with an increase in $d U / d t$, a monotonic increase in $U_{b}$ must be observed. It is this dependence that is obtained when studying breakdown in uniform fields [69, 73-75]. The computational work [76] gives the same result. In the experiment, this increase is, however, slower than linear (1) (in particular, in [75, 77] $U_{b}$ increases as $(d U / d t)^{1 / 2}$ ). This can be explained by the fact that $\tau_{f}$ decreases with increasing voltage [69]. In a long tube, the breakdown voltage as a function of $d U / d t$ was measured in [78] for neon and $\mathrm{Ne}-\mathrm{Ar}$ and $\mathrm{Ar}-\mathrm{Hg}$ mixtures. The results of these measurements are presented in Fig. 17. These measurements were carried out under external illumination of the tube. Due to this, fluctuations of $U_{b}$ were significantly smaller. This means that, in equality (3), $t_{s} \ll t_{f}+t_{w}$. In the region $d U / d t>1-$

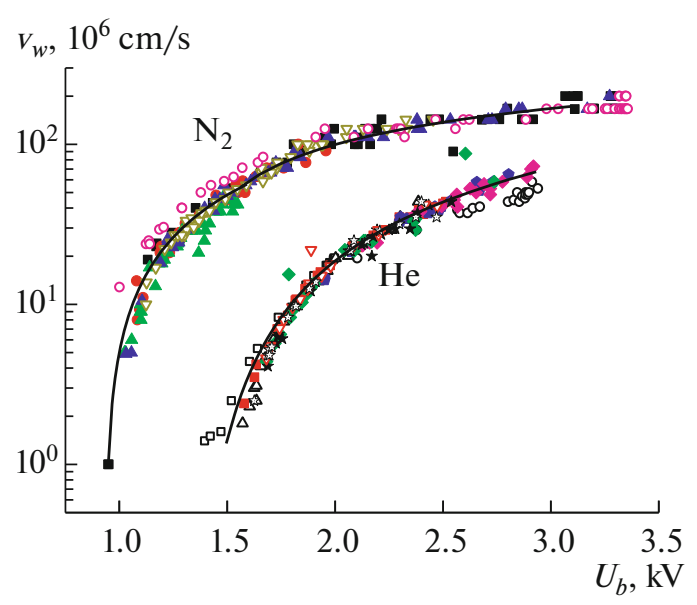

Fig. 16. IW velocity vs. breakdown voltage in nitrogen and helium for $d U / d t=0.5-7.0 \mathrm{kV} / \mathrm{ms}\left(\mathrm{N}_{2}\right)$ and $3-140 \mathrm{kV} / \mathrm{ms}$ (He). Pressure $p=1$ Torr, a tube with a diameter of $d=$ $28 \mathrm{~mm}$ and a length of $l=40 \mathrm{~cm} \mathrm{[70].}$

$10 \mathrm{kV} / \mathrm{ms}$, an increase in $U_{b}$ with an increase in $d U / d t$ is indeed observed, although here it is also slower than linear. This can again be explained by the fact that $\tau_{f}$ decreases with increasing voltage, for long tubes as well [65]. The value of $\tau_{w}$ also decreases with increasing voltage due to an increase in the IW velocity. In any case, however, one can quite confidently assert that the right branch of the curves in Fig. 17 is caused by an increase in the second term of equality (1) with increasing wavefront steepness $d U / d t$. It is more difficult to explain the increase in breakdown voltage with a decrease in the wavefront steepness when it is smaller than $\sim 1 \mathrm{kV} / \mathrm{ms}$. For short gaps, this was not observed in the entire studied range of $d U / d t$, up to very small values: $10^{-2} \mathrm{~V} / \mathrm{s}$ for breakdown in nitrogen [73] and $10^{-1} \mathrm{~V} / \mathrm{s}$ in neon [75].

In [78], the following explanation of the observed regularities was proposed. Under standard conditions of the discharge ignition, before the voltage is applied to the HVE, the entire tube together with the electrodes is at zero potential relative to the ground. When a voltage $U$ is applied to the HVE, all this voltage is applied between the electrode and the wall and the possibility of primary breakdown is determined by the value of $U$ (in particular, a necessary condition for a breakdown is $U>U_{S}$ ). Suppose that some time before the voltage $U$ is turned on, a potential $u_{0}$ is applied to the HVE. If $u_{0}<U_{S}$, this potential will not produce a breakdown, but, due to a finite conductivity of the glass surface and the presence of adsorbed electrons on it, it can increase the wall potential near the HVE, possibly up to $u_{0}$. Then, when a voltage $U$ is applied to the HVE, the potential difference between the electrode and the wall will be smaller than $U$ and, now, the necessary condition for a breakdown will be the 


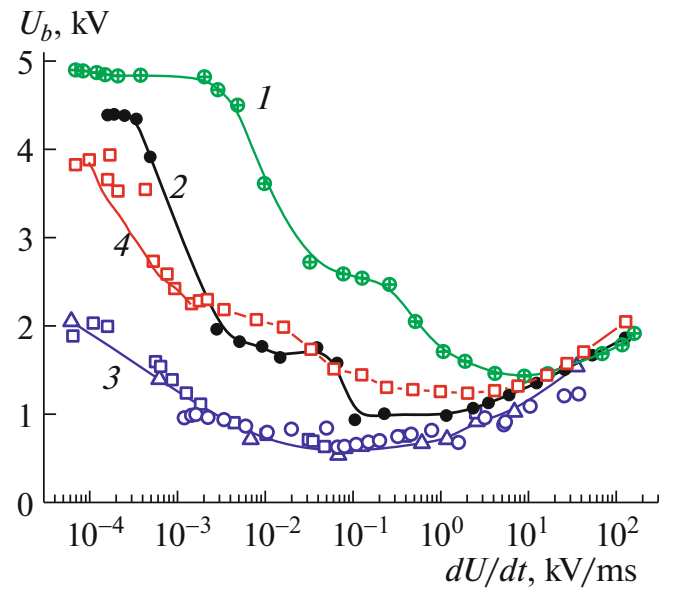

Fig. 17. Breakdown potential vs. anode voltage rise rate in $(1,4) \mathrm{Ne},(2) \mathrm{Ne}-\mathrm{Ar} 3: 1$ mixture, and (3) $\mathrm{Ar}-\mathrm{Hg}\left(p_{\mathrm{Hg}}=\right.$ 1 mTorr) mixture; $p=(1-3) 3$ and (4) 0.6 Torr; $l=80 \mathrm{~cm}$ and $d=(1,2,4) 15$ and (3) $23 \mathrm{~mm} \mathrm{[78].}$

inequality $U-u_{0}>U_{S}$. Thus, the breakdown voltage will increase by $u_{0}$ (or, possibly, by a smaller value if the wall potential does not reach $u_{0}$ ). To verify this assumption, an experiment was conducted in which pulses with a linearly growing wavefront were superimposed on a constant voltage $u_{0}$ and the breakdown voltage was measured for various $u_{0}$. The results are represented by dots in Fig. 18. The straight lines in this graph depict the functions

$$
U_{b}=U_{b}^{0}+u_{0},
$$

where $U_{b}^{0}$ is the value of $U_{b}$ at $u_{0}=0$. It can be seen that, in all cases, the breakdown voltage increases by an amount close to $u_{0}$.

Returning to the results presented in Fig. 17, it should be noted that, for the region of $d U / d t$ to the left of the minimum, the time interval from the beginning of the voltage growth to the breakdown is from $\sim 10 \mathrm{~ms}$ to almost $100 \mathrm{~s}$. It may be assumed that (i) during this time, the wall potential in the anode region also grows, although slower than the potential of the anode itself and (ii) the lower the voltage rise rate, the smaller the lag of the wall potential from the anode potential. A breakdown will occur when the potential difference between them exceeds $U_{S}$ (the contribution of the second term in equality (1) for small $d U / d t$ is insignificant). As a result, with a decrease in $d U / d t$, the breakdown voltage increases. In this case, the actual value of the static breakdown voltage is higher than the nominal value of $U_{S}$ corresponding to the absence of charge on the wall. Thus, in contrast to the right branch of the curves in Fig. 17, the left branch can be qualitatively explained by the increase in the first term of equality (1) with a decrease in the wavefront steepness.

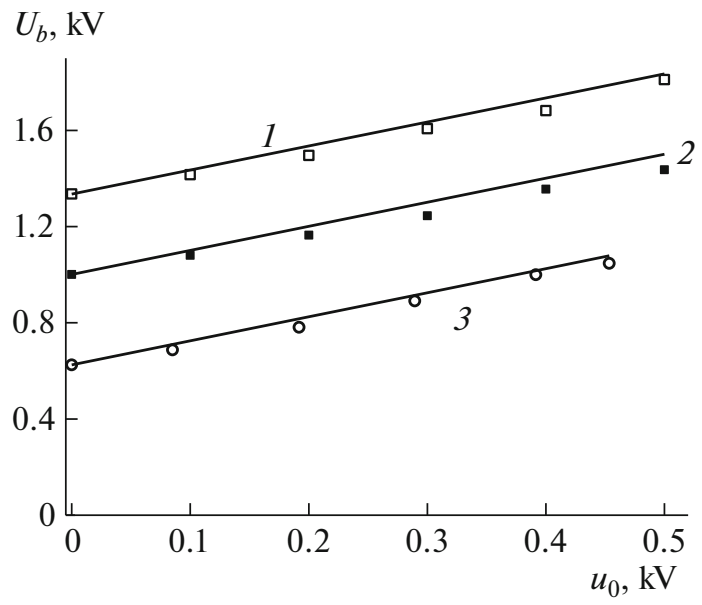

Fig. 18. Breakdown voltage vs. constant bias potential in (1) Ne at $\mathrm{p}=0.6$ Torr, (2) Ne-Ar (3:1) mixture at 3 Torr, and (3) Ar at 1 Torr; $d U / d t=(1,2) 1$ and (3) $0.05 \mathrm{kV} / \mathrm{ms}$; $d=(1,2) 15$ and (3) $28 \mathrm{~mm}$; straight lines: calculation by formula (5) [78].

\subsection{The Memory Effect of the Discharge Gap}

It was said above that the delay of a breakdown can be reduced if there is an additional electron source, e.g., thermionic emission from the high-voltage cathode. The presence of charged or excited particles remaining in the gap after the previous discharge pulse can lead to a similar result. This, in turn, can affect the temporal and other characteristics of the discharge. This kind of "memory effect," apparently discovered for the first time in [79], manifests itself in various situations: during the decay of an arc-discharge plasma [80], the propagation of streamers [81], the motion of an IW in a plasma jet [82], and in nanosecond [83] and barrier [84] discharges. Upon excitation of fast ionization waves, the electrons remaining after the breakdown are the seeds for the generation of the next wave [22]. In a low-pressure glow discharge, this phenomenon was studied by researchers from Serbia in a large series of works, in particular $[69,73-75,77,85]$, in which the time delay $\left(t_{d}\right)$ of the development of a discharge pulse relative to the time at which the voltage applied to the electrodes, depending on interval between pulses, was measured. The Townsend breakdown in a uniform field with an electrode spacing of $\sim 0.1-10 \mathrm{~mm}$ in nitrogen and noble gases at a pressure of $\sim 1-10$ Torr was studied. For small intervals between pulses, $\tau \sim 0.1 \mathrm{~s}$ and shorter, $t_{d}$ was $\sim 100 \mu \mathrm{s}$. With an increase in $\tau$, it increases, and this increase continued up to $\tau \sim 10^{3}-10^{4} \mathrm{~s}$, where $t_{d}$ reached $\sim 10-100 \mathrm{~s}$. Thus, the "memory" of the previous discharge could be preserved for dozens of minutes. Since such a long lifetime of free electrons in a gas is excluded, it should be assumed that electrons appear in the post-discharge phase due to reactions involving long-lived particles. The authors suggest two such reactions, both occurring on the electrode surfaces: ion-electron emission 
and recombination of nitrogen atoms, accompanied by the ejection of an electron from the metal (it is assumed that nitrogen is always present in the gas as an impurity).

Obviously, at a finite rise rate of the interelectrode voltage, a decrease in the breakdown delay must lead to a decrease in the breakdown potential, according to relationship (1). This effect during breakdown in long tubes was studied in [86] (nitrogen), [87, 88] (argon), and [89] (argon with an admixture of nitrogen). Figure 19 shows the voltage waveforms at the high-voltage anode during breakdown in nitrogen in a tube $40 \mathrm{~cm}$ long and $28 \mathrm{~mm}$ in diameter. The pulse front is linear, and $d U / d t=5.1 \mathrm{kV} / \mathrm{ms}$. The voltage pulses were grouped in pairs with a repetition period of $220 \mathrm{~ms}$ and a variable interval between pulses inside a couple $\tau=$ 0.5-64 ms. Breakdown occurs at the point of maximum $U$. It can be seen from the figure that the breakdown voltage in the second pulse of the pair changes with $\tau$ and, for the most part, it is lower than in the first. Figure 20 presents the results of similar measurements in argon. The repetition period of pairs is $2 \mathrm{~s}$. The dots represent the result of averaging over 50 pulses. It can be seen that, at $\tau \approx 500 \mathrm{~ms}$ and smaller, the value of $U_{b}$ for the second pulse is significantly smaller than the average value for the first pulse and is close to its minimum value (the region $\tau<20 \mathrm{~ms}$ will be considered separately). This, obviously, indicates a sharp decrease in the breakdown delay time, up to the complete suppression of the statistical component $t_{s}$. The latter can also inferred from the disappearance of the scatter of $U_{b}$ in the region $\tau<300 \mathrm{~ms}$. In $[86,88]$, estimates are given that imply that the observed effect in nitrogen can be explained by the processes on the electrode surface and the tube wall, suggested in [69]: ion-electron emission and heterogeneous recombination of $\mathrm{N}$ atoms accompanied by electron emission; in argon, they can be explained by the presence of electrons remaining in the anode region after the previous pulse.

It seems obvious that the previous pulse can reduce the breakdown voltage if it ever can affect it. In reality, it follows from Fig. 19 that, for some intervals between pulses (2-3 ms), the breakdown voltage in the second pulse is higher than in the first. We can say that there is an anomalous memory effect. Figure 21 shows what consequences it can have. The oscillograms of voltage and discharge current in nitrogen during breakdown by a periodic sequence of pulses are shown for an interval of $1.7 \mathrm{~ms}$, for which the memory effect is anomalous. The amplitude of the voltage pulse $U_{0}$ is sufficient for the breakdown in the first pulse, but not sufficient for the breakdown in the second. The next (third) pulse is already outside the anomaly zone, and a breakdown is again possible. As a result, a breakdown occurs after one pulse. Such a picture is observed only in a certain range of the pulse frequency. If, without changing $U_{0}$, the frequency is decreased or

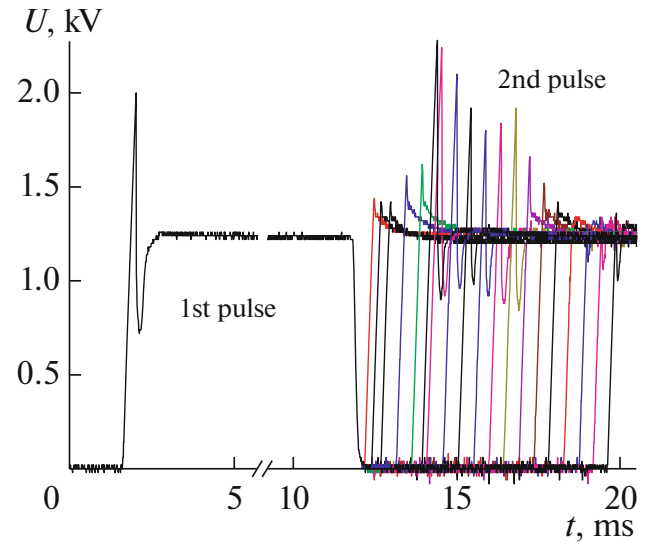

Fig. 19. Time dependences of the anode voltage during breakdown by two pulses with a variable interval between them. Nitrogen, 1 Torr [86].

increased, then the second pulse can also leave the anomaly region and the missed pulses appear.

The manifestation of the anomalous memory effect can also be seen in Fig. 20. In the interval $\tau \approx 2-$ $20 \mathrm{~ms}$, a breakdown in the second pulse occurs at a voltage higher than the minimum value for the first pulse. This effect is more clearly seen in Fig. 22. The difference from Fig. 20 here is that the repetition period of the pulse pairs is reduced to $200 \mathrm{~ms}$. Now the first pulse of the pair falls within the range of the ordinary memory effect with respect to the second pulse of the previous pair and is found in the horizontal section of the lower curve of Fig. 20. For the second pulse in the interval $\tau=1-20 \mathrm{~ms}$, an anomalous effect is observed.

Another anomaly of the effect is that, in the range of its existence and at even smaller $\tau$, no ionization wave before the second pulse is detected. Under the conditions of Fig. 19 (nitrogen), an IW appears starting only with $\tau \approx 2-3 \mathrm{~ms}$, and, under the conditions of Figs. 20 and 22 (argon), starting with $\tau \approx 10-15 \mathrm{~ms}$.

In [86-88], the following explanation of the causes of the anomalous memory effect is suggested. When the duration of the interval between pulses is small, the breakdown in the second pulse occurs under conditions of a high bulk and surface concentration of charged particles remaining after the previous discharge. As a result, the potential of the wall in the near-electrode region is close to the potential of the electrode itself, i.e., there is no potential jump between the high-voltage electrode and the wall, necessary for the primary breakdown and excitation of the ionization wave. On the other hand, the high electron density and the wall charge caused by them concentrate the electric field lines inside the tube and create a longitudinal field in it, as it occurs in a steady-state discharge [3]. In such a field, the discharge can be ignited without an IW, by the ionization multiplication of residual electrons. In this case, the voltage for dis- 


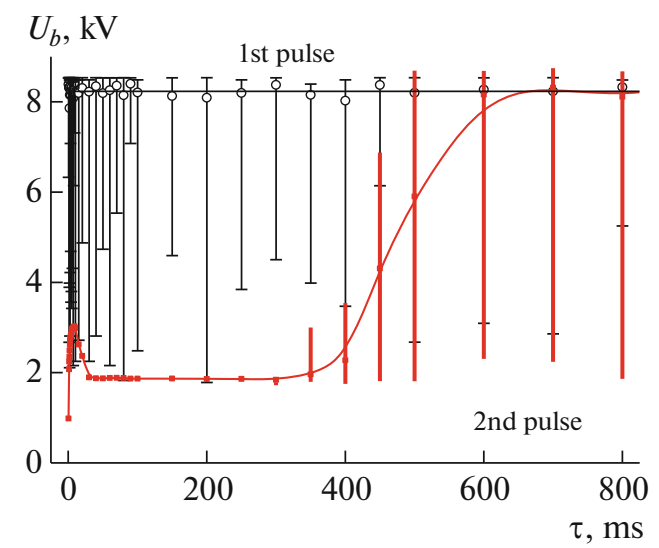

Fig. 20. Breakdown voltages in the 1st and 2 nd pulses of a pair at various intervals between pulses; vertical strokes show boundaries of scatter in a series of 50 measurements. Argon, 5 Torr [88].

charge ignition without the participation of an IW can be higher than that with an IW, because of a less efficient (from the point of view of ionization) potential distribution along the tube.

Note that, in the works [56-58,60], devoted to breakdown in argon or in its mixtures, the pulse repetition rate was $5 \mathrm{~Hz}$. From the data of Figs. 20 and 22, it follows that, under these conditions, a very strong memory effect can be expected.

\subsection{Initiation of Breakdown by Visible Radiation}

In [60], it was mentioned that the external illumination of a discharge tube can change the ignition voltage. Therefore, the shielding of the tube was intended to protect it from this influence as well. Its mechanism was not discussed. The effect on the breakdown of UV, X-ray, and gamma radiation is well understood [8] and its mechanism is clear: this is photoionization of gas or photoelectric effect from the surface of electrodes. For visible radiation, these processes are usually impossible and the mechanism must be different. For the ignition of a low-pressure glow discharge during a Townsend breakdown, there are publications [91-94] by the aforementioned Serbian group. In these studies, the breakdown time delay $t_{d}$ was measured. In [91], a breakdown in neon was studied at a pressure of 10 Torr and the interelectrode gap was illuminated by the radiation of a tunable laser at a wavelength of $614.3 \mathrm{~nm}$, corresponding to the transition $1 s_{5}-2 p_{6}$ in the $\mathrm{Ne}$ atom. In [92-94], a breakdown in nitrogen at a pressure of 1 Torr was studied, and the gap was illuminated with radiation from a nitrogen discharge. In all cases, irradiation changed the delay time, but the sign of this change was different: in [9193], $t_{d}$ increased and, in [94], decreased. In explaining the result, in the first case, an assumption is made that the radiation destroys the metastable states $\mathrm{Ne}\left(1 s_{5}\right)$
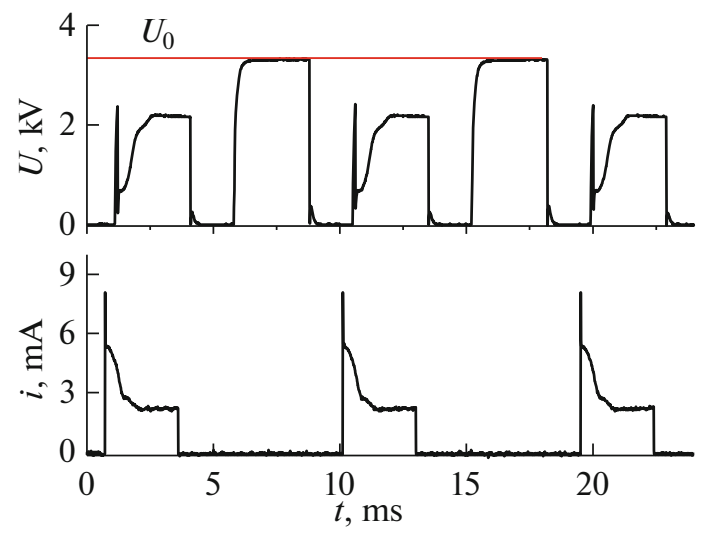

Fig. 21. Time dependences of voltage and current during breakdown in nitrogen by periodic pulses; $U_{0}$ is the voltage pulse amplitude [90]; $p=1$ Torr.

and $\mathrm{N}_{2}\left(A^{3} \Sigma_{u}^{+}\right)$and thereby reduces the rate of ionization processes occurring with their participation, which leads to an increase in $t_{d}$. The authors of [94] relate their result to photoelectric effect from the cathode surface. They had to assume the presence on the cathode surface of a microfilm of iron oxide with a longer photoelectric threshold wavelength than that of the metal.

In long tubes, the effect of illumination by visible light on the breakdown was observed in [95-97]. The source of irradiating light was a tungsten halogen lamp with a power of $150 \mathrm{~W}$. Standard fluorescent lamps were studied, which contained an $\mathrm{Hg}-\mathrm{Ar}$ mixture at a pressure of $150-500 \mathrm{~Pa}$ and had a length of $50 \mathrm{~cm}$ and a diameter of $28 \mathrm{~mm}$ without the fluorescent coating. In [97], the lamp was installed in a metal housing with an aperture for irradiation with light. Voltage pulses had the shape

$$
U=U_{0}[1-\exp (-t / \tau)]
$$

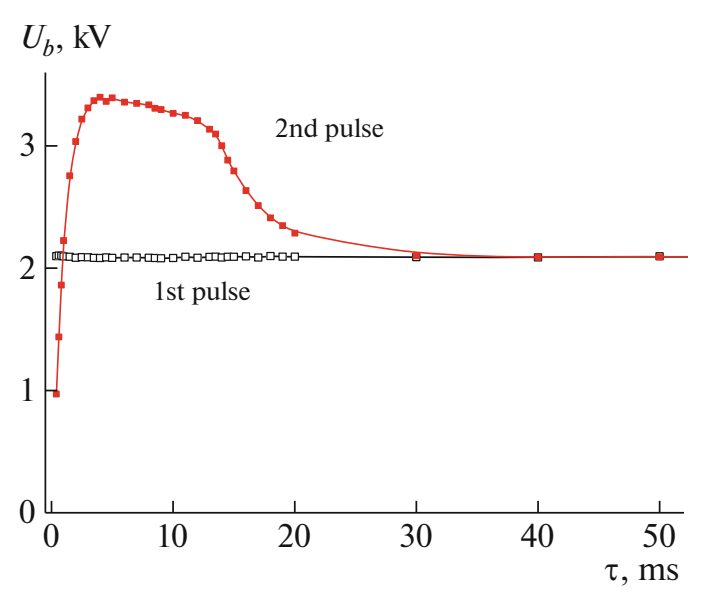

Fig. 22. Breakdown voltages in the 1 st and 2 nd pulse of a pair at different intervals between pulses. The repetition period of pairs is $200 \mathrm{~ms}$. Argon, 5 Torr [87]. 
with $\tau \approx 10 \mathrm{~ms}$ and a repetition rate of $10 \mathrm{~Hz}$. The value of $U_{0}$ was chosen so as the discharge ignition occurred once every $2-3$ seconds. The authors define this a value as the ignition voltage; obviously, it correlates (but does not coincide) with the breakdown voltage. An increase in $U_{0}$ under illumination was observed; this effect intensified with increasing light intensity and, for illuminance of $\sim 10^{3}$ lux, it saturated, reaching almost 1.5 times. For pulses of negative voltage, it was significantly weaker. A study of the spectral dependence of the effect showed that there is a long-wavelength cutoff about $500 \mathrm{~nm}$. It turned out that the effect depends on the position of the irradiated area along the tube: it weakened away from the anode, but, under certain conditions, the dependence was nonmonotonic, with a maximum at a distance of several centimeters from the anode (Fig. 23). The authors suggested two possible mechanisms for the effect of radiation on the discharge ignition: 1) radiation destroys metastable levels of argon, thereby suppressing the Penning ionization of $\mathrm{Hg}$ atoms and 2) it changes the state of the surface charge of the wall and, as a result, affects the motion of the ionization wavefront.

In $[65,71,88,98]$, the effect of illumination on the dynamic breakdown voltage $U_{b}$ in long tubes filled with $\mathrm{He}[71,98], \mathrm{Ar}[71,88,98], \mathrm{Ne}$, or an $\mathrm{Ar}-\mathrm{Hg}$ mixture $[65,98]$ at a pressure of $1-5$ Torr was studied. The breakdown was initiated by high voltage pulses with a steepness of $d U / d t$ up to $\sim 10 \mathrm{kV} / \mathrm{ms}$ or with a leading edge described by Eq. (6) with $\tau \approx 50 \mu$ s. Tubes with a diameter of $15-28 \mathrm{~mm}$ were illuminated by filtered radiation of a halogen lamp or LEDs. In contrast to the results of [95-97], illumination in all cases reduced the average value of $U_{b}$ by a factor of up to 2-3. In addition, the statistical scatter of $U_{b}$ decreased or almost vanished. A long-wavelength cutoff of the effect of about 400-500 nm was found. In some cases, as in [95], the points most sensitive to illumination were located at a distance of 5-6 cm from the anode. At a greater distance of the illuminated region from the anode, in particular, when illuminating the near-cathode region, the effect disappeared.

Some of the results are shown in Figs. 24 and 25. Figure 24 shows the dependence of the breakdown voltage in helium and argon on the intensity of illumination of the anode region by the halogen lamp used as a light source. The intensity was varied using neutral filters. There is a pronounced saturation of this dependence, in which the value of $U_{b}$ decreases by a factor of $2-3$. At the same time, its scatter is reduced by a large factor.

Figure 25 shows how the breakdown voltage changed when the anode region of the tube was illuminated by LEDs emitting at different wavelengths. The LEDs had a power consumption of $\approx 50 \mathrm{~mW}$. For one of the points in this graph $(\lambda 532 \mathrm{~nm})$, a laser diode with a power input of $\approx 500 \mathrm{~mW}$ was used. Again, a decrease in $U_{b}$ by a factor of 1.5-2 and a sharp

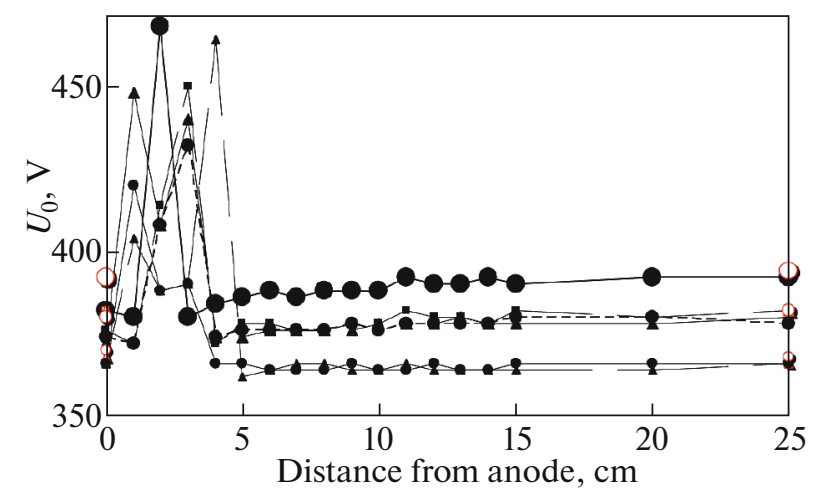

Fig. 23. Discharge ignition voltage under illumination of the tube at different points and at different parameters of the voltage pulse; white dots: in darkness. Fluorescent lamp, $\mathrm{Ar}-\mathrm{Hg}$ mixture, $p=350 \mathrm{~Pa}$ [97].

decrease in its scatter are observed. The long-wavelength cutoff of this effect is seen. Based on these results, it lies in the range $490-530 \mathrm{~nm}$.

In [72], the effect of ullumination on the ignition of a pulsed discharge in a flow through discharge tubes with an internal diameter of $1-4 \mathrm{~mm}$ in $\mathrm{He}, \mathrm{N}_{2}$, and $\mathrm{O}_{2}$ and in $\mathrm{He}-\mathrm{N}_{2}, \mathrm{He}-\mathrm{O}_{2}$ mixtures was studied. The light sources were LEDs, lasers, and a xenon lamp; the spectral range from 400 to $660 \mathrm{~nm}$ was studied. The gas pressure varied in the range $2-90 \mathrm{kPa}$, and the flow rate was $0.5-4 \mathrm{~L} / \mathrm{min}$. High voltage pulses with a duration of $2 \mu \mathrm{s}$ were applied to external electrodes placed at a distance of $18 \mathrm{~mm}$ from each other. The breakdown delay time was measured by the duration of the gap between the voltage pulse and the discharge glow pulse. Its value without illumination ranged from a few seconds for helium to more than 5 min for nitrogen. Under illumination, it decreased by several orders of magnitude: in helium, up to $<100 \mu$ s and, in nitrogen, up to several seconds. The approximate position of the long-wavelength cutoff is $500 \mathrm{~nm}$. The maximum efficiency was achieved when the region near the high-voltage anode was illuminated.

The work [66] is the only one in which the effect of pulsed visible radiation on the breakdown in a long tube was studied. The discharge tube with neon at a pressure of 0.6 Torr had a diameter of $15 \mathrm{~mm}$. The light sources were a LED emitting at a wavelength of $460 \mathrm{~nm}$ and a laser diode with a radiation wavelength of $407 \mathrm{~nm}$. They operated in a pulsed mode with a variable pulse duration of $0.1-100 \mu \mathrm{s}$. A region of the tube with a diameter of $5 \mathrm{~mm}$ was illuminated in the immediate vicinity of the high-voltage anode or at a distance of $5 \mathrm{~cm}$ from it towards the cathode. The input radiation power was $\approx 0.03 \mathrm{~mW}$ and $\sim 10 \mathrm{~mW}$ for the LED and the laser, respectively. The voltage pulse at the anode of the tube was either rectangular with a leading edge duration of about $1 \mu \mathrm{s}$ or had a linearly growing edge with a slope of $\approx 1-100 \mathrm{kV} / \mathrm{ms}$. The pulse repetition period was $1 \mathrm{~s}$ or more in order to minimize 


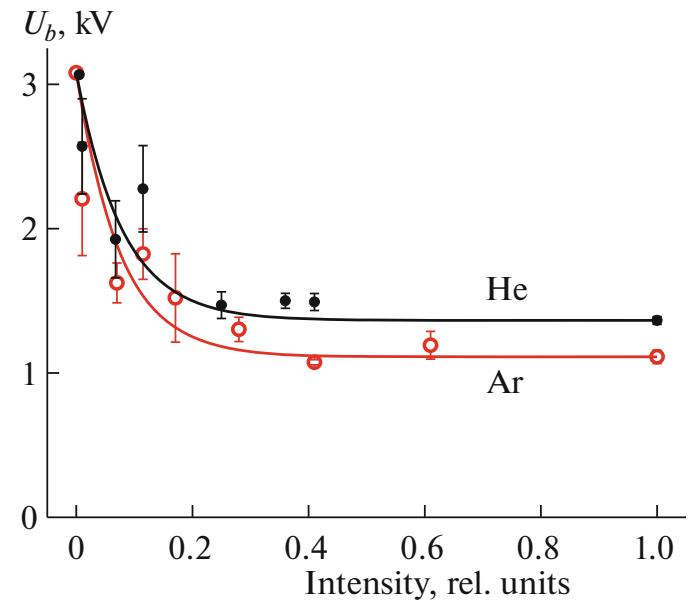

Fig. 24. Mean breakdown voltage vs. radiation intensity of illuminating halogen lamp; $p=1$ Torr [71].

the memory effect. With a rectangular pulse, the breakdown time delay $t_{d}$ was measured. Figure 26 shows the measurement results for 300 consecutive pulses in darkness and when illuminating the anode region with a LED. Radiation pulses of duration $\delta t=$ $5 \mu$ s were delayed relative to the leading edge of the voltage pulse by $t_{0}=0.3 \mathrm{~ms}$. As can be seen from the figure, in the absence of illumination, $t_{d}$ had a very wide scatter: from $20 \mu$ s to $1 \mathrm{~ms}$. A light pulse limits this scatter from above by $t_{0}$. Near the time $t_{0}$, there is a condensation of points, since all breakdowns that, without illumination, should have occurred after $t_{0}$ occur at $t \approx t_{0}$. With decreasing $t_{0}$, the boundary becomes lower. It turned out that the effect is observed even if the light pulse goes ahead of the voltage pulse wavefront by $\left|t_{0}\right| \leq 150 \mathrm{~ns}$. With an increase in the duration of the light pulses, the picture in Fig. 26 did not change, but, with its decrease, the effect weakened, the upper boundary of the scatter spread, and, at $\delta t \leq 0.2 \mu \mathrm{s}$, the effect disappeared.

For pulses with a linearly growing wavefront, the breakdown potential $U_{b}$ was measured. Figure 27 shows the results of such measurements for three series of 50 successive voltage pulses with a wavefront steepness of $5 \mathrm{kV} / \mathrm{ms}$ in darkness (a) and upon illumination with the LED of a region $5 \mathrm{~cm}$ away from the anode $(\mathrm{b}$, c). Light pulses with a duration of $10 \mu$ s were delayed relative to the moment of the onset of voltage growth by $t_{0}=0.15$ or $0.4 \mathrm{~ms}$. The heavy shorter lines represent the voltage at the moment of the light pulse, $U\left(t_{0}\right)$, and the thin longer lines are drawn through the points with the minimum $U_{b}=U_{\min }$, which is the same for all three cases. Light pulses reduce the scatter of points, and the effect is most noticeable when the delay $t_{0}$ is equal to or slightly smaller than $t^{*}$, where $t^{*}$ is such that $U\left(t^{*}\right)=$ $U_{\text {min }}$. In this case, the scatter practically disappears and $U_{b}=U_{\min }$. For $t_{0}>t^{*}$, all breakdowns occur in the interval between $U_{\min }$ and $U\left(t_{0}\right)$.

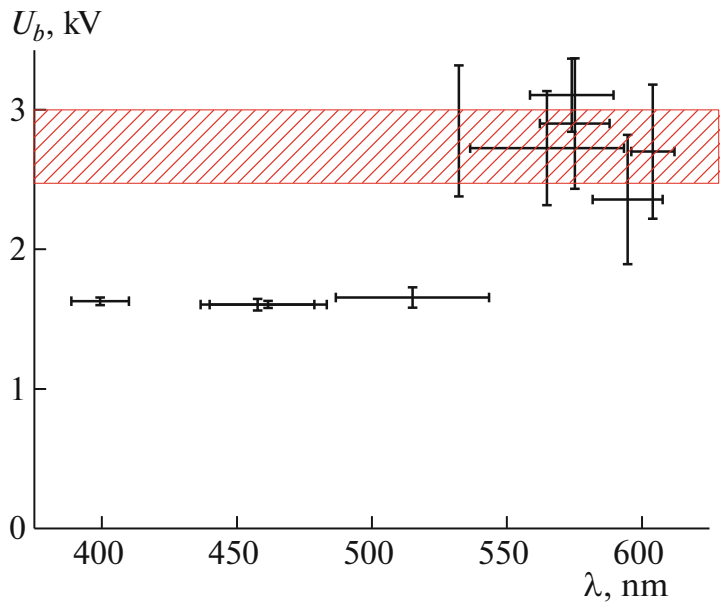

Fig. 25. Breakdown voltage in $\mathrm{Ne}(p=0.6$ Torr) when the tube is illuminated by radiation of different wavelengths; shaded area shows breakdown voltage without illumination [65]. (C) IOP Publishing. Reproduced with permission. All rights reserved.

Obviously, the cause of the observed effects in all cases shown in Figs. 24-27 should be sought in the behavior of the breakdown time delay $t_{d}$, more precisely, its statistical component $t_{s}$. The value of $t_{s}$ is determined by the production rate of "effective" electrons (formula (3)). The question of possible mechanisms of electron emission under irradiation was discussed in $[65,66,71,72,88]$. The authors of [71] and then of [72] considered as such a mechanism optically stimulated exoelectronic emission (OSEE). This is a special case of the so-called exoelectronic emission: the emission of electrons from the surface of a body upon preliminary excitation by external action [99]. In [71, 72], it was assumed that such excitation could occur upon contact of the wall with the plasma of the previous discharge pulse-an assumption not supported by any justification. Earlier, OSEE was suggested as a mechanism of the influence of visible light on the ignition of a corona discharge in a tip-plane gap [100]. In that case, there was evidence that the

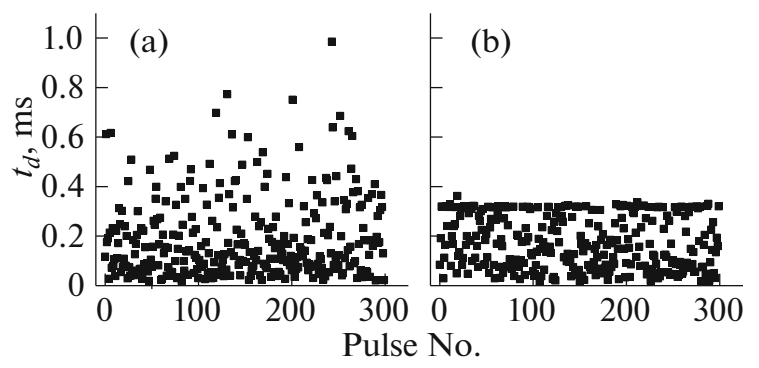

Fig. 26. Breakdown time delay for a series of 300 pulses (a) in darkness and (b) under illumination. The light pulse is delayed relative to the voltage pulse wavefront by $0.3 \mathrm{~ms}$. Ne, 0.6 Torr [66]. (C) IOP Publishing. Reproduced with permission. All rights reserved. 


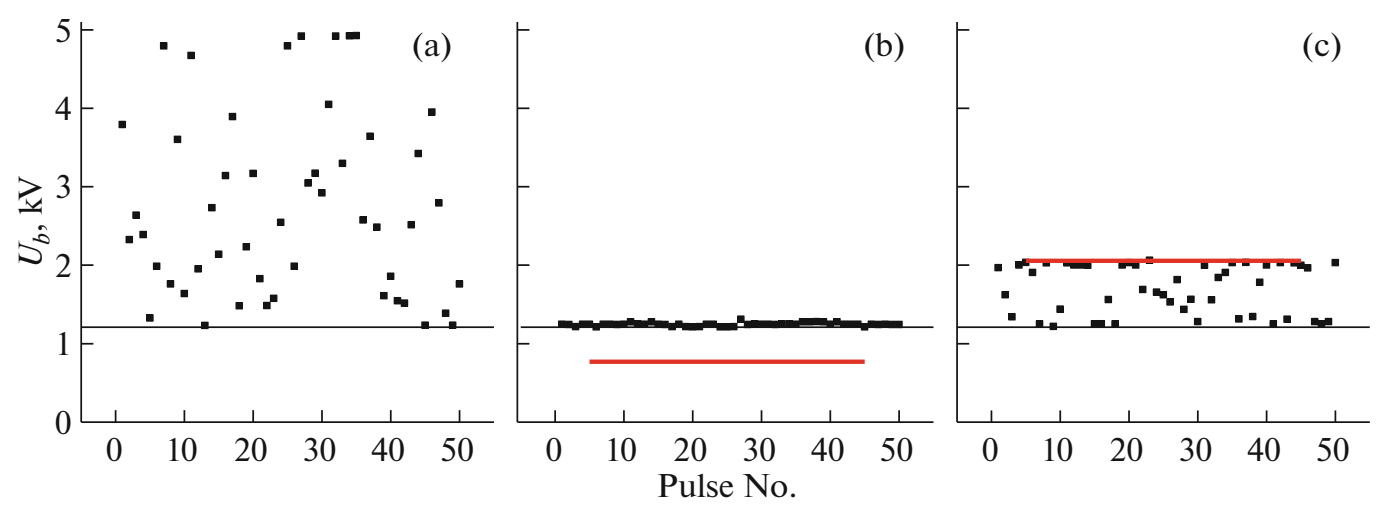

Fig. 27. Breakdown voltage for a series of 50 pulses (a) in darkness and (b, c) under illumination. The light pulse is delayed relative to the onset of voltage growth by (b) 0.15 and (c) $0.4 \mathrm{~ms}$. Ne, 0.6 Torr, and $d U / d t=5 \mathrm{kV} / \mathrm{ms}$ [66]. (C) IOP Publishing. Reproduced with permission. All rights reserved.

excitation really occurred; it was caused by mechanical surface treatment. In [72], vibrational excitation of $\mathrm{N}_{2}$ molecules under illumination was also considered as a mechanism of the action on a breakdown, which had to lead to a decrease in the ionization threshold (nitrogen was present as a component of the mixture or as an impurity). In reality, such a mechanism is excluded, because optical transitions between the vibrational levels of $\mathrm{N}_{2}$ are forbidden.

In $[65,66,88]$, another mechanism of electron emission was considered, namely, their photodesorption from the wall surface. It is considered established that this process occurs in dielectric barrier discharges (DBD) at atmospheric pressure [101, 102]. In the work [103], devoted to the modeling of a DBD in nitrogen in the Townsend mode, it was shown that the model leads to adequate results after including into it electron desorption from the dielectric surface. It is assumed that the adsorbed electrons are bound to the surface by polarization forces with a relatively low binding energy $(\sim 1 \mathrm{eV})$. In [102, 104, 105] it was experimentally found that electrons adsorbed on the surface of a dielectric in a barrier discharge can remain there after the cessation of plasma exposure for several minutes or even hours. It is important that desorption of electrons can occur under the action of photons. In [105], illumination of a dielectric (BSO crystal) with a LED at a wavelength of $632 \mathrm{~nm}$ reduced the surface charge lifetime by a factor of $10^{3}$. In [106], the ignition voltage of barrier discharge in helium decreased significantly when a dielectric (glass) was irradiated with a neodymium laser at a wavelength of $\lambda 532 \mathrm{~nm}$, and radiation with $\lambda 1064 \mathrm{~nm}$ caused a transition of a discharge from the glowing mode to Townsend. It is believed that it is the desorption of electrons upon illumination of a dielectric surface with discharge radiation that leads to the synchronization of breakdowns in the filament form of barrier discharge [101, 102, 104]. Thus, it is possible that it is the process of photodetachment of electrons adsorbed on the surface of a discharge tube that is the mechanism of the influence of visible radiation on the breakdown in long tubes. Another confirmation of this fact is that such an influence is observed only with a positive pulse polarity. Irradiation of the region near the high-voltage cathode (with a grounded anode) does not have an effect. Indeed, an electron emitted by the wall is found in the repulsive electric field of the cathode and cannot participate in ionization processes.

\subsection{Breakdown in a Tube with an Ungrounded Electrode}

In Section 4.1, we talked about the need to ground the low-voltage electrode. It was assumed that both electrodes are connected to the outputs of the current source (Fig. 7). In this section, we will consider the case in which one of the electrodes remains unconnected to the power circuit (Fig. 28). Such a scheme was used in [46]. It is obvious that a steady-state discharge in this case is not possible. However, the primary breakdown between the HVE and the wall and the IW motion proceed without the participation of the second electrode. Therefore, they must proceed in the usual way. The fact that the state of the low-voltage electrode does not affect the propagation of a fast IW is noted in the literature [15, 19, 28]. In [107], this was verified for a pre-breakdown IW. The measurements were carried out in a tube with a diameter of $15 \mathrm{~mm}$ and a length of $80 \mathrm{~cm}$. The tube contained a $\mathrm{Ne}-\mathrm{Ar}$ mixture with a concentration ratio of $3: 1$ (the socalled K4 mixture, often used in lighting engineering) at a pressure of 1 Torr. The breakdown was initiated by positive or negative rectangular pulses with a duration of $500 \mathrm{~ms}$ and a repetition period of $1 \mathrm{~s}$.

Figure 29 shows the results of measurements in a tube with an ungrounded cathode (heavy lines). For comparison, similar curves are shown for a conventional connection scheme, in which the cathode is grounded, i.e., connected to the power source. It can be seen that, even with an ungrounded cathode, a primary breakdown (time $t_{1}$ ) is observed, accompanied by a jump in voltage and current. But the cathode cur- 


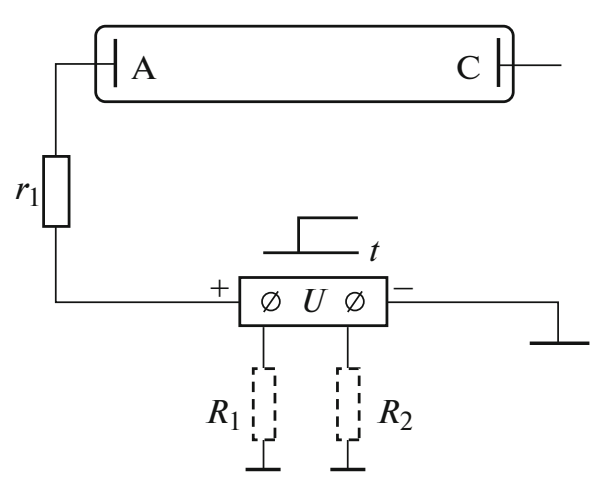

Fig. 28. Schematics of connecting the tube with a nongrounded cathode.

rent (time $t_{2}$ ) in this case does not appear, and the anode voltage and current return to the initial level and then do not change. Simultaneously with the primary breakdown, a radiation pulse arises from the anode region and an IW, moving toward the cathode, appears. It follows from the figure that, on the interval $t_{1}-t_{2}$, i.e., since the initial breakdown to the breakdown of the entire tube, or, in other words, the whole time of the IW motion, the anode current and voltage with the grounded and ungrounded cathode are the same. A similar picture was observed with a negative pulse polarity. Figure 30 shows the dependence of the IW velocity on the distance to the high-voltage cathode for a grounded (1) and non-grounded (2) anode. It can be seen that the velocities coincide over the entire gap, except for the last section, near the anode. In view of the aforesaid, the results of Figs. 29 and 30 seem to be predictable.

The following effect was unexpected. After the end of the voltage pulse, after a certain (random) period of time, surges were again observed on the curves of the anode current and voltage, but of the reverse sign, compared to Fig. 29: a surge up in the voltage and a surge down in the current. This is illustrated by Fig. 31a. (The appearance of short spikes of current marked with asterisks at the leading and trailing edges of the pulse is associated with recharging of the capacitance of the anode and connected circuit elements relative to the ground.) A similar effect was observed during breakdown by pulses of negative polarity (Fig. 31b). Voltage and current jumps, now at the cathode, are visible both during the initial breakdown and after the end of the pulse.

The observed picture was explained as follows. The primary breakdown charges the wall, and, after the breakdown, its potential near the high-voltage electrode is approximately equal to the electrode potential. For some time, the wall charge is preserved. After the pulse terminates and the electrode potential becomes equal to zero, a potential difference between the electrode and the wall appears, which is approximately the same as in the beginning of the pulse, but of the oppo-

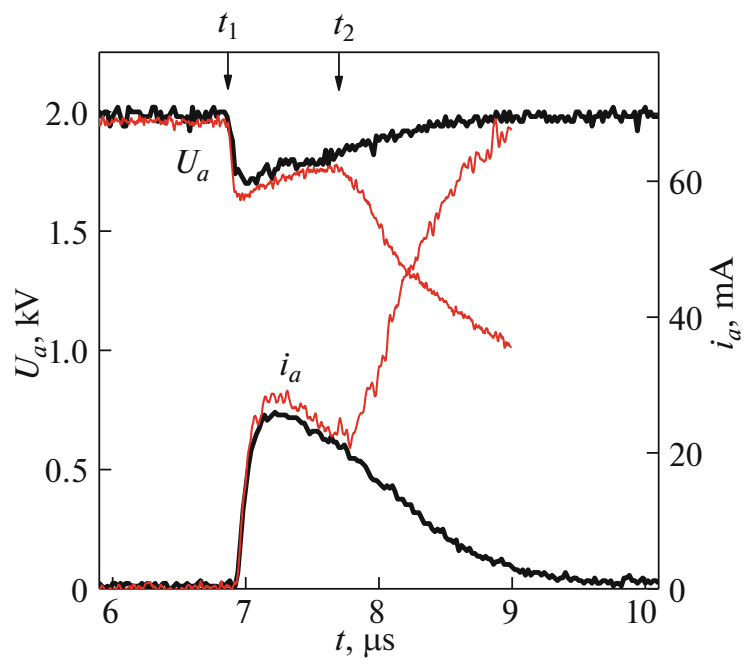

Fig. 29. Time dependences of the anode voltage and anode current during breakdown of a tube with (heavy lines) a non-grounded and (thin line) grounded cathode. $\mathrm{Ne}-\mathrm{Ar}$ (3:1) mixture, 1 Torr [107].

site sign. This leads to a breakdown between the wall and the electrode, the same as the primary breakdown, but with the opposite sign. In [107], this breakdown was called reverse. Ionization waves initiated by this breakdown were also observed. They were detected by their radiation. Curves in Fig. 32 show the time profile of the brightness of the IW radiation from the points of the tube located at different distances from the high-voltage electrode. For all graphs, the first curve on the left corresponds to a point directly above the HVE; the next, to a point at a distance of $10 \mathrm{~cm}$ from it, etc.; and the last one, to a point above the second (free) electrode. Graphs in Figs. 32a and $32 \mathrm{~b}$ were plotted for the positive voltage polarity, and the graphs in Figs. 32c and Figs. 32d, for the negative

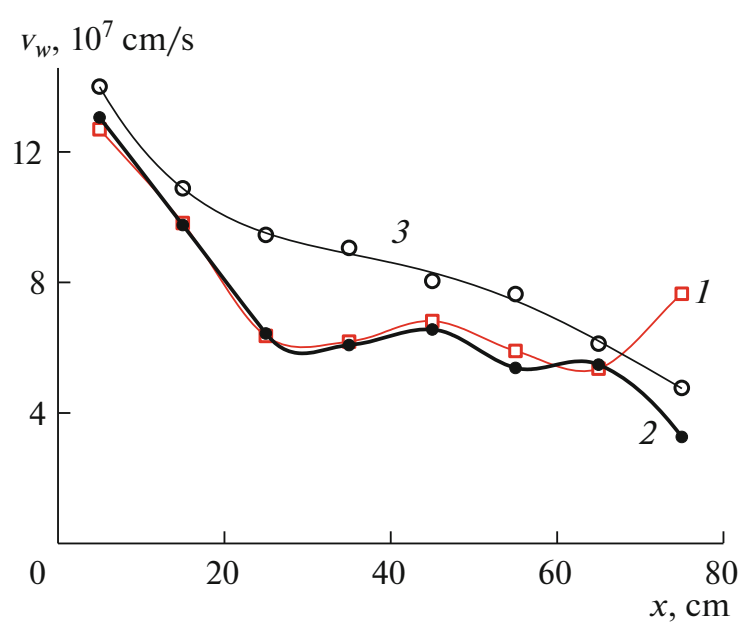

Fig. 30. IW velocity vs. distance from the cathode for (1) a grounded and $(2,3)$ non-grounded anode; (3) reverse breakdown wave [107]. Conditions of Fig. 29. 

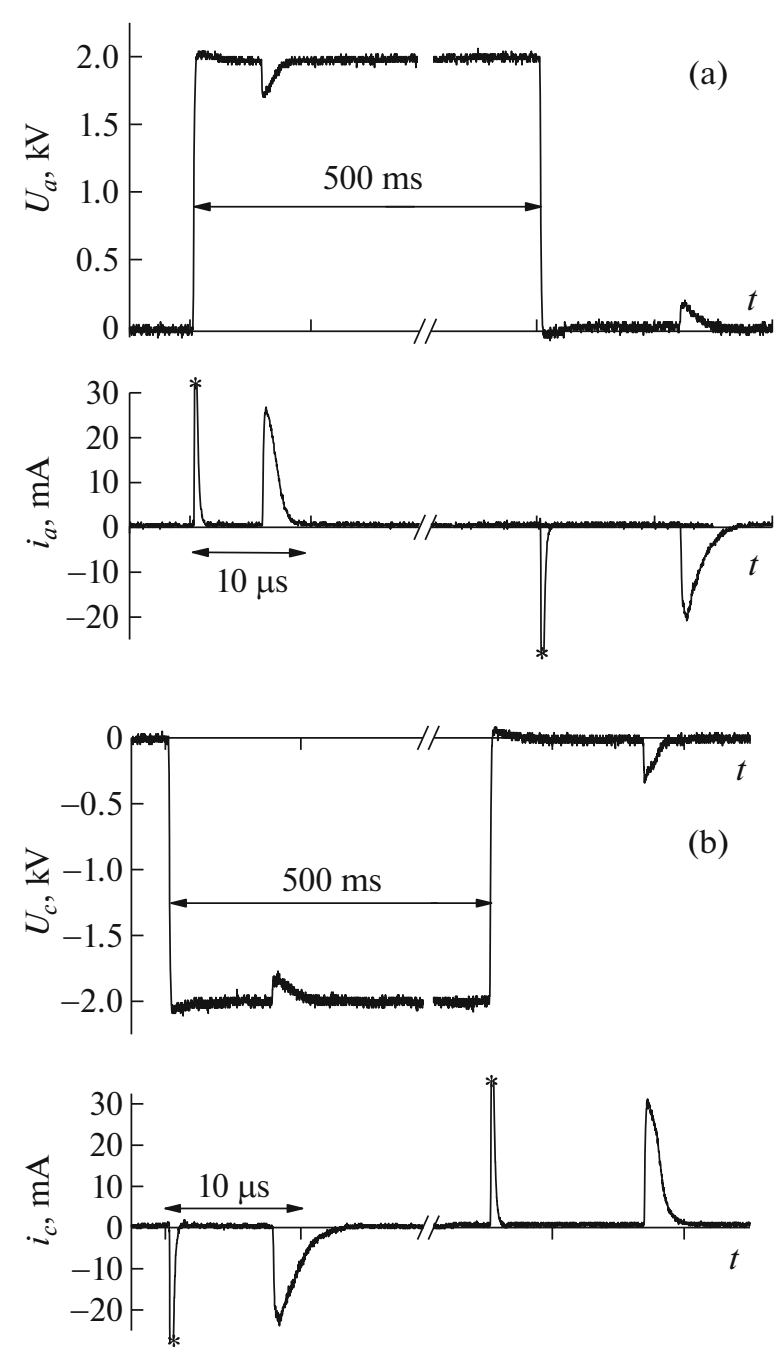

Fig. 31. Time dependences of voltage and current of the high-voltage electrode during breakdown by a pulses of (a) positive and (b) negative polarity; the second electrode is non-grounded; asterisks indicate current spikes caused by recharging the capacitance of the HVE with connected circuit elements [107]. Conditions of Fig. 29.

polarity. The upper curves $(\mathrm{a}, \mathrm{c})$ refer to the primary breakdown, and the lower curves (b, d), to the reverse breakdown. From the arrangement of the curves, it follows that the IW moves from the high-voltage electrode to an ungrounded one for both the primary and the reverse breakdown.

In Fig. 30, curve 3 represents the dependence of the velocity of the IW of the reverse breakdown on the longitudinal coordinate for the negative pulse polarity; it is somewhat higher than for the IW of the primary breakdown. With a positive polarity, the velocity ratio is opposite. Since, for the primary breakdown, the velocity of the positive wave is higher than that of the negative wave, it turns out that the velocities of the waves of the reverse breakdown for the positive polarity and of the primary breakdown for the negative polarity are almost the same. The same is true for

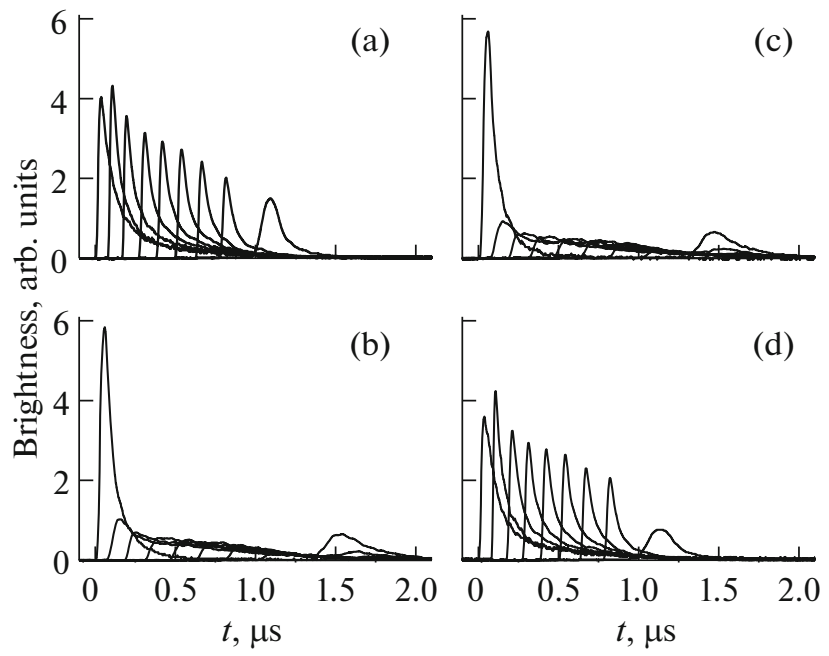

Fig. 32. Time profile of the brightness of the radiation of an IW, recorded at various distances $(0,10,20, \ldots, 80 \mathrm{~cm})$ from the HVE for $(a, b)$ positive and $(c, d)$ negative polarity of the voltage pulse; $(a, c)$ primary and $(b, d)$ reverse breakdown [107]. Conditions of Fig. 29.

another pair: the reverse breakdown of negative polarity and the primary breakdown of positive polarity. The same cross symmetry is seen in Fig. 32.

The propagation of an IW is accompanied by charging the tube wall. The value of the charge was measured by integrating the voltage drop across the ballast resistance. The results obtained for both types of waves and voltage polarities are presented in Fig. 33 . It follows from the figure that the absolute value of the charge is the same within several percent for the primary and reverse breakdown, regardless of the pulse polarity. Hence, the wall charge did not change between the primary and reverse breakdown and, during the reverse breakdown, the wall, charged in the primary breakdown, was completely neutralized. In particular, this implies that, if a high voltage is applied to the electrode before the reverse breakdown occurs, the next breakdown will not occur, while the same pulse applied after this moment would lead to a breakdown. An experiment confirming this conclusion was conducted. To the high-voltage electrode, a pair of pulses with an interval of $10 \mu$ s between them was supplied. The mean delay time of the reverse breakdown was comparable with this interval. It turned out that the second pulse produced a breakdown only if, during this time, the reverse (in relation to the first pulse) breakdown had time to occur.

The ionization wave observed in [107] during a reverse breakdown differs from the return wave that often accompanies the breakdown of ordinary, grounded tubes [35, 36, 55-60]. The return wave (return stroke) moves from the low-voltage electrode to the high-voltage one, i.e., in the direction opposite to the wave of the primary breakdown. Its velocity is usually much higher than the velocity of the forward 


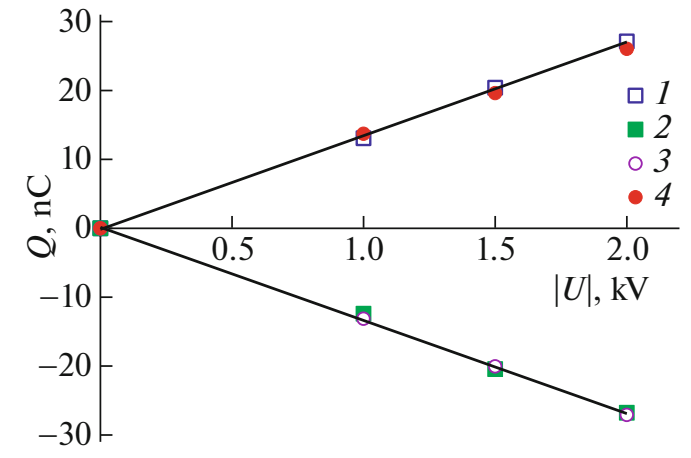

Fig. 33. Charge carried by the ionization wave in $(1,3)$ a primary and $(2,4)$ reverse breakdown for $(1,2)$ positive and $(3,4)$ negative polarity at different pulse amplitudes [107]. Conditions of Fig. 29.

wave. The return wave removes the charge from the wall, but only partially [60]. In the given case, both waves moved in the same direction with close velocities and the wave of the reverse breakdown completely removed the wall charge left by the wave of the primary breakdown.

\subsection{Radiation Spectrum of an Ionization Wave}

In a pre-breakdown ionization wave, a high electric field strength is realized. In particular, in [60], the values obtained in argon reach $\approx 200 \mathrm{~V} / \mathrm{cm}$, which corresponds to a reduced field strength $E / N \approx 200 \mathrm{Td}$. This is approximately 50 times greater than in the positive column of a steady-state discharge under similar conditions [108]. As a result of this difference, the radiation spectra of an IW and a steady-state discharge must differ very strongly. In the spectrum of the wave, lines or bands from the high levels should be clearly visible, including the levels of ions, which, usually, are hardly visible or not visible at all in the positive column of a glow discharge. This is actually observed in the experiments. For example, Fig. 34 shows oscillograms of the emission brightness of the bands of the nitrogen ion and molecule during a breakdown in a tube $40 \mathrm{~cm}$ long and $28 \mathrm{~mm}$ in diameter with a pulse of positive polarity [109]. Narrow brightness peaks correspond to the radiation from the IW; here, the ionic peak is two times brighter than the molecular one. After $0.35 \mathrm{~ms}$, discharge radiation begins; here, the ionic luminescence is orders of magnitude weaker than the molecular luminescence. This is not surprising, since the excitation energy $\left(E^{*}\right)$ of the level $\mathrm{N}_{2}^{+}\left(B^{2} \Sigma_{u}^{+}, v^{\prime}=0\right)$ is almost two times higher than that of the level $\mathrm{N}_{2}\left(C^{3} \Pi_{u}, v^{\prime}=0\right)$ (19 and $11 \mathrm{eV}$, respectively). From the intensity ratio of the bands of $\mathrm{N}_{2}^{+}$and $\mathrm{N}_{2}$, it was possible to estimate the electric field strength in the IW. This estimate gave $E / N \approx 900 \mathrm{Td}$, while, in the positive discharge column, $E / N=80 \mathrm{Td}$.

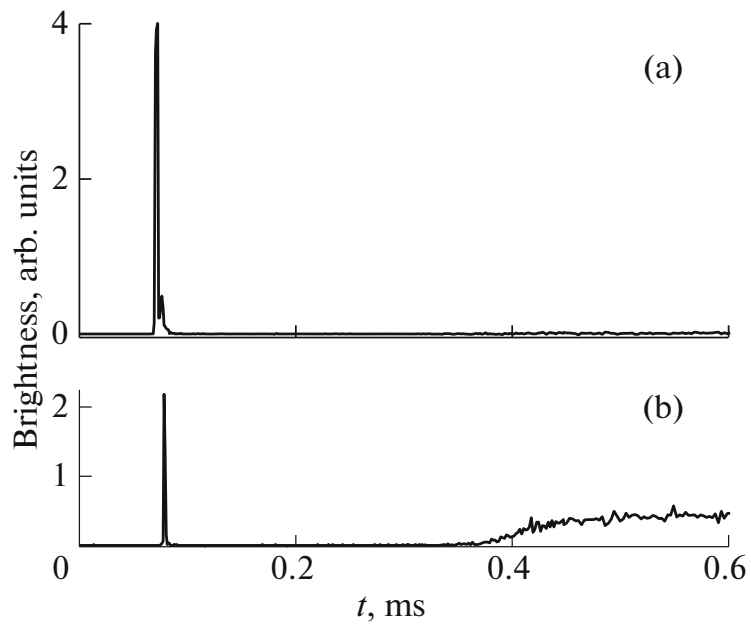

Fig. 34. Oscillograms of radiation brightness of the bands (a) $\mathrm{N}_{2}^{+}\left(B^{2} \Sigma_{u}^{+} \rightarrow X^{2} \Sigma_{g}^{+}, 0-0\right)$ and (b) $\mathrm{N}_{2}\left(C^{3} \Pi_{u} \rightarrow B^{3} \Pi_{g}\right.$, $0-2)$ during breakdown by a pulse of positive polarity in nitrogen, $p=1$ Torr [109].

Figure 35 shows the brightness records of the spectral lines of radiation by an IW from the anode region during breakdown by a positive voltage pulse of the $\mathrm{Ne}-\mathrm{Ar}(3: 1)$ mixture at a pressure of 1 Torr [107]. The emission spectrum of a steady-state discharge contained mainly lines of atomic argon $\left(E^{*} \approx 13 \mathrm{eV}\right)$; the neon lines $(18.5 \mathrm{eV})$ were hardly visible, and the ion lines with $E^{*}=32 \mathrm{eV}\left(\mathrm{Ar}^{+}\right)$and $56 \mathrm{eV}\left(\mathrm{Ne}^{+}\right)$were not visible at all. In the spectrum of the IW, all these components were detected near the high-voltage electrode for both positive and negative polarity. With increasing distance from the HVE, the $\mathrm{Ne}^{+}$lines disappeared, but only they. A similar picture was observed for the reverse breakdown wave, and there was a cross symmetry similar to that observed for the integral intensity (Fig. 32): the spectral distribution in the IW of the primary breakdown at positive polarity repeated the distribution of the IW of the reverse breakdown of negative polarity, and vice versa.

Figure 36 shows similar results for an $\mathrm{Ar}-\mathrm{Hg}$ mixture $\left(p_{\mathrm{Ar}}=3\right.$ Torr, $\left.p_{\mathrm{Hg}}=1 \mathrm{mTorr}\right)$ in a tube $23 \mathrm{~mm}$ in diameter and $80 \mathrm{~cm}$ long [63]. The spectrum of the steady-state discharge in this mixture consisted only of mercury lines, which is not surprising due to the very low position of the levels of the $\mathrm{Hg}$ atom $\left(E^{*} \approx 5\right.$ and $8 \mathrm{eV})$. At the same time, the most pronounced in the IW were the argon lines, and the $\mathrm{Ar}^{+}$lines were detected with confidence. However, in a negative IW, the $\mathrm{Ar}^{+}$lines were not detected.

Having data on the relative brightness of two lines with very different excitation energies, it is possible to calculate the electric field strength in which the excitation occurs. Such a non-invasive method (in contrast to the probe method in [60]) is used to diagnose plasma of short-duration discharges. In particular, it 


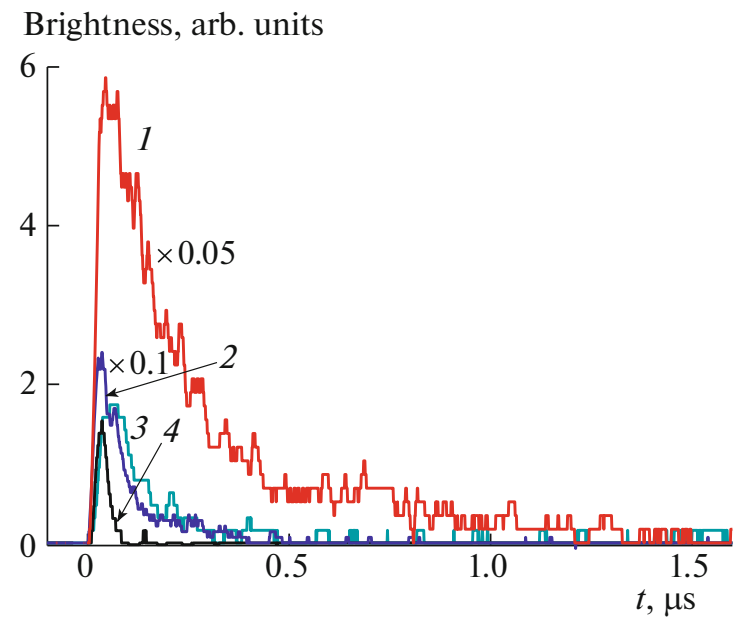

Fig. 35. Time dependences of radiation brightness of the lines: (1) Ar $\lambda 763.5 \mathrm{~nm}$, (2) $\mathrm{Ne} \lambda 640.2 \mathrm{~nm}$, (3) $\mathrm{Ne}^{+}$ $\lambda 450.8 \mathrm{~nm}$, and (4) $\mathrm{Ar}^{+} \lambda 459.0 \mathrm{~nm}$ in a positive IW in a $\mathrm{Ne}-\mathrm{Ar}$ mixture, $p=1$ Torr [107].

was used to measure the field strength in nanosecond discharges (FIW) in nitrogen [110] and helium [111]. In [112], a model was created that made it possible to apply this approach to the diagnosis of a pre-breakdown IW. With its help, the experimental data presented in Fig. 36, as well as the data obtained for other points of the discharge gap, were processed. Two pairs of lines were used as analytic: $\operatorname{Ar}^{+}(488.0 \mathrm{~nm}) / \operatorname{Ar}(763.5$ $\mathrm{nm})$ and $\operatorname{Ar}^{+}(488.0 \mathrm{~nm}) / \mathrm{Hg}(546.1 \mathrm{~nm})$. The results obtained satisfactory agreed with each other (Fig. 37). The nonmonotonic dependence of the voltage on the axial coordinate was also observed in [60]. For a negative wave, the values of $E / N$ turned out to be approximately half that, which also agrees with [60]. For comparison, it can be noted that, in a steady-state discharge, $E / N \approx 1.2$ Td [63].

\subsection{The Discharge after the Passage of an Ionization Wave}

The pre-breakdown wave carries out the initial ionization, i.e., leaves a plasma behind it. Further development and reaching the characteristics of a steadystate discharge proceeds in various processes of ionization and electron removal and can be rather complicated. Therefore, the time dependences of the electrical and optical characteristics of the discharge after the passage of an IW and before the establishing of steady-state parameters can have a sophisticated form. An example may be the oscillograms of the anode voltage $U_{a}$ and cathode current $i_{c}$ during a breakdown in a $\mathrm{He}-\mathrm{Ne}$ mixture at a pressure of 4 Torr (Fig. 5) [51]. At the time $t_{1}$, a primary breakdown occurred, and, at the time $t_{2}$, a breakdown of the entire gap arose and a current in the cathode circuit appeared. Then,

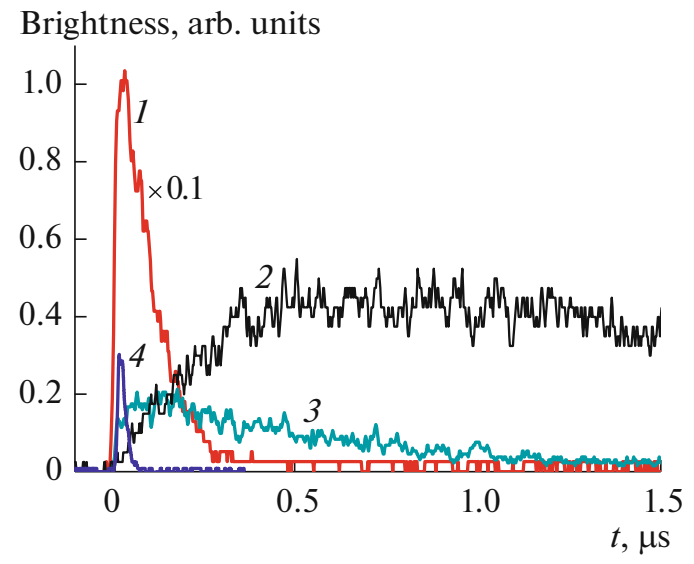

Fig. 36. Time dependences of radiation brightness of the lines: (1) $\mathrm{Ar} \lambda 763.5 \mathrm{~nm}$, (2) $\mathrm{Hg} \lambda 253.7 \mathrm{~nm}$, (3) $\mathrm{Hg}$ $\lambda 546.1 \mathrm{~nm}$, and (4) $\mathrm{Ar}^{+} \lambda 488.0 \mathrm{~nm}$ in a positive IW in an $\mathrm{Ar}-\mathrm{Hg}$ mixture; $p_{\mathrm{Ar}}=3$ Torr and $p_{\mathrm{Hg}}=1$ mTorr [63].

for $50 \mu \mathrm{s}$, the anode voltage was zero, and, only after that, the values of $U_{a}$ and $i_{c}$ reached the steady-state level. The discharge radiation during these $50 \mu \mathrm{s}$ had a strongly nonmonotonic time dependence.

Figures 38 and 39 show the results of studies of the discharge ignition in a tube $3 \mathrm{~cm}$ in diameter and $40 \mathrm{~cm}$ long in $\mathrm{He}-\mathrm{N}_{2}$ mixtures with a positive polarity of the voltage pulse [113]. The tube was U-shaped, and optical measurements were carried out along the axis of the horizontal part, where the discharge positive column was located. The time scale of the measurements did not make it possible to see a detailed picture of the initial stage of breakdown. The current waveform in Fig. 38 shows that, on the scale of the graph,

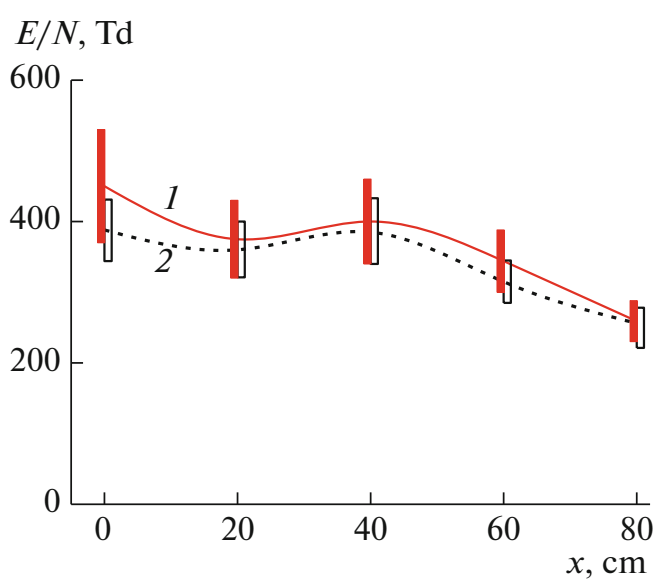

Fig. 37. The values of $E / N$ in a positive IW at different distances from the anode, obtained from the line intensity ratios (1) $\mathrm{Ar}^{+}(488.0 \mathrm{~nm}) / \mathrm{Ar}(763.5 \mathrm{~nm})$ and (2) $\mathrm{Ar}^{+}$ $(488.0 \mathrm{~nm}) / \mathrm{Hg}(546.1 \mathrm{~nm})$ [112]. Ar- $\mathrm{Hg}$ mixture, $p=$ 3 Torr, $p_{\mathrm{Hg}}=1$ mTorr. 


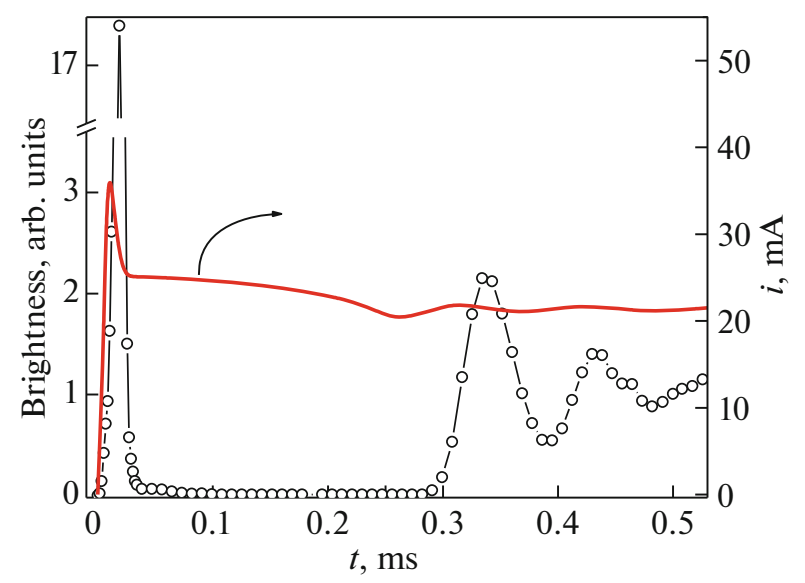

Fig. 38. Time dependences of radiation brightness of the $\mathrm{N}_{2} \lambda 337.1 \mathrm{~nm}$ band and the discharge current during breakdown in a $\mathrm{He}+0.2 \% \mathrm{~N}_{2}$ mixture, $p=5$ Torr [113].

the breakdown occurred almost at the pulse wavefront and then the current changed insignificantly until reaching a steady-state value. But, at the same time, for $0.3 \mathrm{~ms}$, the radiation of the molecular nitrogen band $\lambda 337.1 \mathrm{~nm}$ was more than two orders of magnitude weaker than in the steady-state discharge (in the terminology of [113], the "dark phase" of the development of the discharge was observed). The radiation brightness of other $\mathrm{N}_{2}$ bands and all $\mathrm{He}$ lines behaved in a similar way. This could be explained only by the fact that the field strength in the positive column in this time interval is small. This was confirmed by probe measurements (solid curve in Fig. 39). The following explanation of this picture is given in [113]. Immediately after the breakdown, the discharge current is small and the entire voltage of the power source drops in the discharge gap, which leads to a very large value of $E / N$. In this field, metastable helium levels are intensely excited. This leads to the formation of an excess amount of electrons generated in the Penning ionization reaction

$$
\mathrm{He}^{*}+\mathrm{N}_{2} \rightarrow \mathrm{He}+\mathrm{N}_{2}^{+}+e
$$

( $\mathrm{He}^{*}$ is a metastable helium atom). In turn, this leads to a redistribution of the source voltage between the discharge and the ballast resistor and the field in the discharge falls below the steady-state level. In this field, the rate of diffusion loss of electrons is small; as a result, the lifetime of a weak field can significantly exceed the time of the decay of metastable states to the steady-state level. This was confirmed by measuring the He* number of density (Fig. 39). In [113], calculations confirming the correctness of this model were carried out.

In pure noble gases, the analog of reaction (7) is the process

$$
\mathrm{A}^{*}+\mathrm{A}^{*} \rightarrow \mathrm{A}+\mathrm{A}^{+}+e
$$

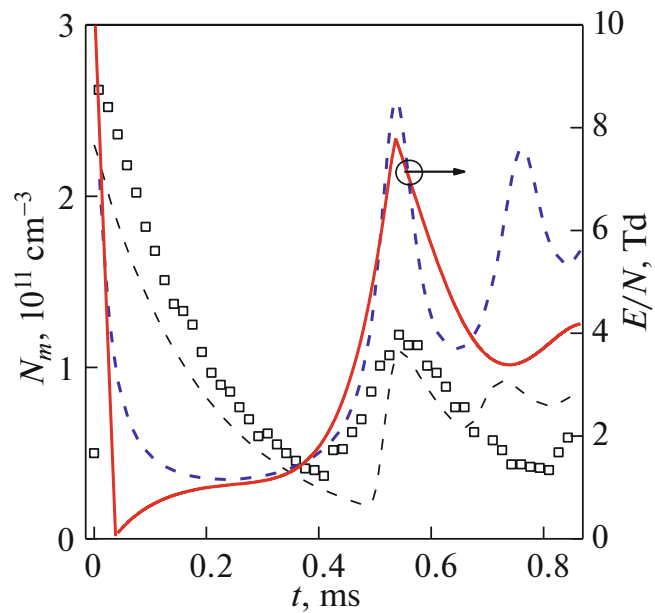

Fig. 39. Reduced electric field strength (solid curve) and number of density of $\mathrm{He}^{*}\left(2^{3} S_{1}\right)$ atoms (dots) during breakdown in a $\mathrm{He}+0.02 \% \mathrm{~N}_{2}$ mixture, $p=5$ Torr; dashed curves: calculation [113].

where $A^{*}$ is an atom in a metastable state. Therefore, one could expect the appearance of the dark phase there too. Indeed, it was found in helium in the same work [113] at a pressure of 5 Torr, and then in argon in a tube with a diameter of $27 \mathrm{~mm}$ and a length of $30 \mathrm{~cm}$ at a pressure of 5 Torr [114] and in neon in a tube with a diameter of $4.5 \mathrm{~cm}$ and a length of $33 \mathrm{~cm}$ at a pressure of 10-15 Torr [115]. The duration of the interval with a strongly reduced glow intensity was $1-1.5 \mathrm{~ms}$, and, in helium, it reached $2.5 \mathrm{~ms}$. A necessary condition for this effect was a sufficiently long interval between pulses and a large ballast resistance in the discharge circuit. Both these factors made it possible to ignite a discharge under strong overvoltage, which was necessary for a large initial value of $E / N$. Models based on the assumption of the decisive role of process (8) satisfactorily described the experiment. A picture similar in physical nature and manifestation was also observed under other conditions: in a continuous discharge in helium after additional excitation of the plasma by a high-voltage nanosecond pulse [116, 117].

The reaction of the form (8) is also known for nitrogen:

$$
\mathrm{N}_{2}^{*}\left(A^{3} \Sigma_{u}^{+}, a^{\prime 1} \Sigma_{u}^{-}\right)+\mathrm{N}_{2}^{*}\left(a^{\prime 1} \Sigma_{u}^{-}\right) \rightarrow \mathrm{N}_{4}^{+}+e,
$$

where $A^{3} \Sigma_{u}^{+}$and $a^{\prime 1} \Sigma_{u}^{-}$are metastable states. It could therefore be assumed that a similar effect must also occur in nitrogen. It was indeed obtained in [118] (Figs. 40 and 41). A peak of the current and glow at the leading edge of the pulse was observed, followed by a dip of the anode voltage and glow for $0.2 \mathrm{~ms}$. The depth of the dip in the radiation brightness reached three orders of magnitude relative to the steady-state level. Thus, the picture looked the same as in noble gases. However, it was not possible to explain it in a 

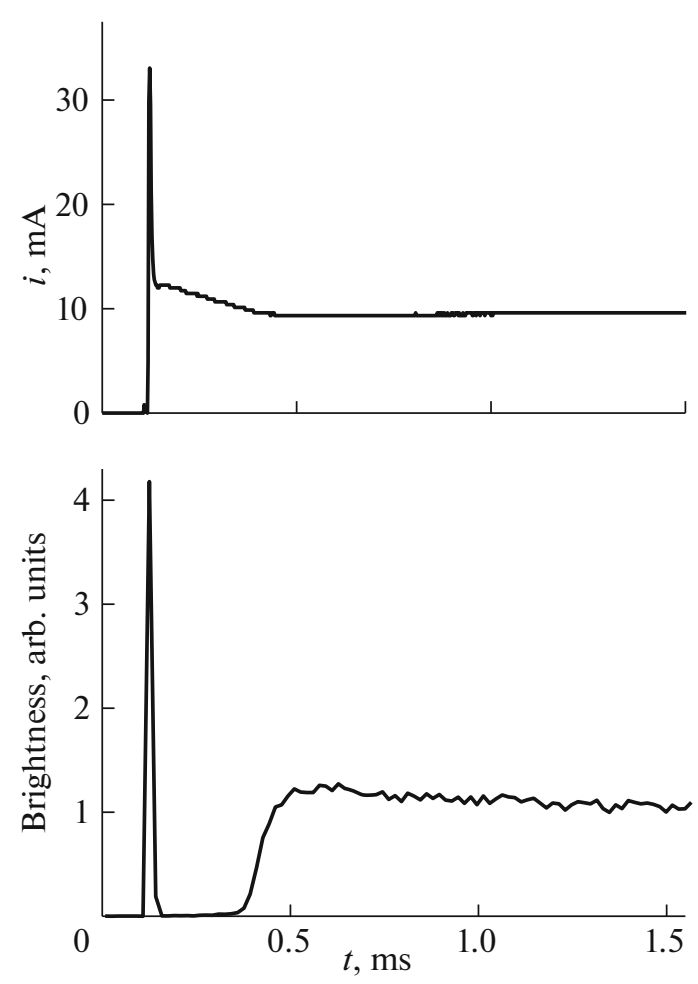

Fig. 40. Time dependences of the discharge current and brightness of the $\mathrm{N}_{2} \lambda 337.1 \mathrm{~nm}$ band in the beginning of a discharge pulse of positive polarity at $p=1$ Torr [118].

similar way, i.e., involving process (9). The model did not reproduce the nonmonotonic behavior and dips in the curves of the electric field strength, anode voltage, and brightness. The reason was an "unfavorable" ratio between the rate constants of reaction (9) and excitation of metastable states of $\mathrm{N}_{2}^{*}$, as a result of which, in the initial surge of the field strength, a peak of $\mathrm{N}_{2}^{*}$ concentration noticeably exceeding the steady-state level is not formed. More detailed experiments showed that the initial peak of the glow brightness does not occur synchronously along the tube length, but moves from the anode to the cathode with a velocity of $\sim 10^{7} \mathrm{~cm} / \mathrm{s}$. Thus, in fact, this peak was the radiation of a pre-breakdown ionization wave. If we assume that the density of electrons created by the wave is sufficiently high, then the conditions for the appearance of the dark phase can be produced. In the model of [118], this was simulated by imposing nonzero initial conditions for the density of charged particles. By adjusting the initial density, it was possible to obtain results close to experimental (Fig. 41). An additional confirmation of the determining role of the IW in creating the dark phase effect was that it was much weaker with a negative polarity of the voltage pulse. In this case, the radiation of a negative IW was also so weak that it could be detected only near the cathode [109].
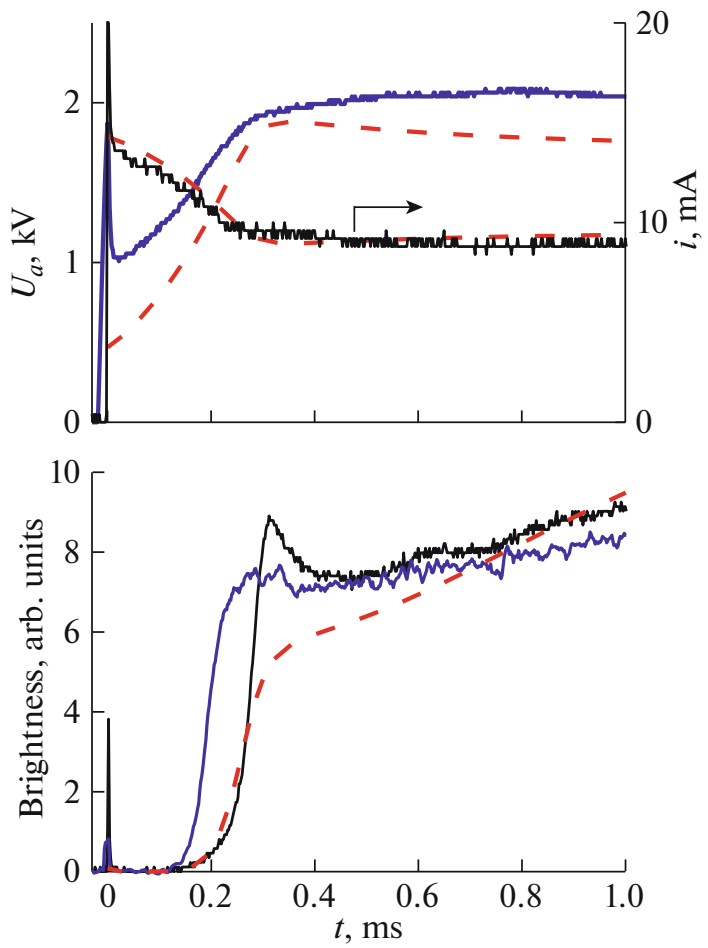

Fig. 41. Time dependences of the anode voltage, discharge current, and brightness of the integral emission from two different points of the discharge; dashed curves: calculation. Nitrogen, 1 Torr [118].

\section{CONCLUSIONS}

Analysis of publications concerning the processes and mechanisms of ignition of a glow discharge in tubes whose length significantly exceeds their diameter ("long" discharge tubes) at reduced pressures $(\sim 10$ Torr and below) and moderate voltage rise rates $(d U / d t \sim 1 \mathrm{kV} / \mu \mathrm{s}$ and smaller) leads to the following conclusions. The electric field in such tubes before the breakdown is substantially nonuniform. Therefore, a breakdown occurs after a pre-breakdown ionization wave passes the discharge gap. In turn, an IW arises during the initial breakdown between the high-voltage electrode (HVE) and the nearby section of the tube wall. The velocity of the IW ranges from $\sim 10^{5}$ to $\sim 10^{7} \mathrm{~cm} / \mathrm{s}$. During its motion to the low-voltage (grounded) electrode, the IW charges the tube wall.

The properties of an IW depend on the polarity of the HVE. Therefore, the main characteristics of the breakdown-the breakdown potential and the delay time-depend on which of the electrodes, the anode or the cathode, are under the high voltage. The breakdown voltage, in addition to the type of gas, depends in a complex way on the geometry of the discharge gap and cannot be quantitatively described by any universal regularity. The development of breakdown is largely affected by the processes involving the wall of the discharge tube. This, in particular, is manifested in the character of the dependence of the breakdown poten- 
tial on the rise rate of the applied voltage, in the possibility of initiating a breakdown by external illumination by visible radiation, and in the existence of a reverse breakdown in a tube with an insulated electrode.

All this makes the breakdown processes in long tubes significantly different from the breakdown between large-area closely spaced electrodes, where the electric field is uniform before the breakdown and where the classical Townsend (avalanche) or, under strong overvoltage, streamer mechanism is realized. On the other hand, the nature of these processes is very different from that of nanosecond discharges arising at voltages with a steepness $d U / d t \sim 1 \mathrm{kV} / \mathrm{ns}$ and higher and associated with fast $\left(\sim 10^{9} \mathrm{~cm} / \mathrm{s}\right)$ ionization waves (FIWs). At the wavefront of a FIW, runaway electrons, which play a significant role in its formation, are generated. The properties of FIWs significantly differ from the properties of slow pre-breakdown IWs considered above. A high steepness of the voltage pulse is a necessary condition for the generation of FIWs, and their velocity increases with increasing steepness. Slow IWs can be generated at any wavefront steepness, and their velocity does not depend explicitly on $d U / d t$. If a FIW passes through a preionized gas, its velocity increases with increasing electron density. On the contrary, the generation of a slow wave can be hampered and even blocked by residual electrons, which causes an anomalous memory effect. Finally, the dielectric walls of the tube, although they can influence the propagation of FIWs, do not play a key role.

From the analysis of publications it also follows that a number of issues are currently not fully clarified. These include, e.g., the mechanism of generation of seed electrons necessary for the propagation of positive IWs. There are serious contradictions between the data of various authors on the effect of external illumination on the breakdown potential (according to some data, it decreases, and, according to others, increases). It is unclear why, in some cases, the point most sensitive to illumination is located at a certain distance from the high-voltage anode. It is not quite clear how the additional potential applied to the electrode propagates along the surface of the wall, affecting the breakdown voltage. Most of the experimental works were performed with a shielded discharge tube, and the calculation works also suggest the presence of a shield. In this case, the question of the possible role of the electric effect of the shield on the breakdown process is omitted.

In conclusion, it should be noted that slow IWs can be of interest themselves, regardless of the breakdown process. For example, in [119], the "collision" of two such waves moving towards each other was investigated. (Earlier, similar processes of interaction of waves and their merging and splitting were studied concerning the FIWs [120, 121].)

\section{FUNDING}

This work was supported by the Russian Foundation for Basic Research, project no. 19-02-00288.

\section{OPEN ACCESS}

This article is distributed under the terms of the Creative Commons Attribution 4.0 International License (http://creativecommons.org/licenses/by/4.0/), which permits unrestricted use, distribution, and reproduction in any medium, provided you give appropriate credit to the original author(s) and the source, provide a link to the Creative Commons license, and indicate if changes were made.

\section{REFERENCES}

1. H. B. O. Davis, Electrical and Electronic Technologies: a Chronology of Events and Inventors to 1900 (Scarecrow Press, Metuchen, NJ, 1981).

2. Spectroscopy of Gas-Discharge Plasma, Ed. by S. E. Frish (Nauka, Leningrad, 1970) [in Russian].

3. Yu. P. Raizer, Gas Discharge Physics (ID Intellekt, Dolgoprudnyi, 2009; Springer, Berlin, 1997).

4. A. Javan, W. R. Bennett, and D. R. Herriott, Phys. Rev. Lett. 6, 106 (1961).

5. J. S. Townsend, Electricity in Gases (Clarendon Press, Oxford, 1915).

6. L. B. Loeb, Fundamental Processes of Electrical Discharges in Gases (Wiley, New York, 1939).

7. M. Mitchner and C. H. Kruger, Jr., Partially Ionized Gases (Wiley, New York, 1973).

8. Electrical Breakdown of Gases, Ed. by J. M. Meek and J. D. Craggs (Wiley, New York, 1978).

9. H. Raether, Electron Avalanches and Breakdown in Gases (Butterworths, London, 1964).

10. Yu. D. Korolev and G. A. Mesyats, Physics of Pulsed Gas Breakdown (Nauka, Moscow, 1991) [in Russian].

11. R. Seeliger, R. Bock, Z. Phys. 110, 717 (1938).

12. W. Bartholomeyczeyk, Ann. Phys. 36, 485 (1939).

13. I. S. Marshak, Sov. Phys.-Usp. 3, 624 (1961).

14. K. Takashima, I. V. Adamovich, Z. Xiong, M. J. Kushner, S. Starikovskaia, U. Czarnetzki, and D. Luggenhölsche, Phys. Plasmas 18, 083505 (2011).

15. L. M. Vasilyak, S. V. Kostyuchenko, N. N. Kudryavtsev, and I. V. Filyugin, Phys.-Usp. 37, 247 (1994).

16. J. J. Thomson, Notes on Recent Researches in Electricity and Magnetism (Clarendon, Oxford (1893), p. 115.

17. J. W. Beams, Phys. Rev. 36, 997 (1930).

18. L. B. Snoddy, J. W. Beams, and J. R. Dietrich, Phys. Rev. 50, 469 (1936).

19. L. B. Snoddy, J. R. Dietrich, and J. W. Beams, Phys. Rev. 52, 739 (1937).

20. F. H. Mitchell and L. B. Snoddy, Phys. Rev. 72, 1202 (1947).

21. P. W. Winn, J. Appl. Phys. 38, 783 (1967).

22. T. Suzuki, J. Appl. Phys. 48, 5001 (1977).

23. R. Kh. Amirov, E. I. Asinovskii, V. V. Markovets, A. S. Panfilov, and I. S. Samoilov, in Low Temperature Plasma, Vol. 9 (Nauka, Novosibirsk, 1994), p. 373 [in Russian]. 
24. L. M. Vasilyak, E. I. Asinovskii, and I. S. Samoilov, in Encyclopedia of Low-Temperature Plasma, Ed. by V. E. Fortov: Introductional Volume (Nauka/Interperiodika, Moscow, 2000), Part II, p. 225 [in Russian].

25. T. B. Anikin, S. A. Bozhenkov, D. V. Zatsepin, E. I. Mintusov, S. V. Pancheshnyi, S. M. Starikovskaya, and A. Yu. Starikovskii, in Encyclopedia of Low-Temperature Plasma, Vol. VIII-1: Chemistry of Low-Temperature Plasma, Ed. by Yu. A. Lebedev, N. A. Plate, and V. E. Fortov (Yanus-K, Moscow, 2005), p. 171 [in Russian].

26. S. M. Starikovskaia, N. B. Anikin, S. V. Pancheshnyi, D. V. Zatsepin, and A. Yu. Starikovskii, Plasma Sources Sci. Technol. 10, 344 (2001).

27. A. N. Lagarkov and I. M. Rutkevich, Electrical Breakdown Waves in Bounded Plasmas (Nauka, Moscow, 1989) [in Russian].

28. A. N. Lagarkov and I. M. Rutkevich, Ionization Waves in Electrical Breakdown of Gases (Springer-Verlag, New York, 1994).

29. L. B. Loeb, Science 148, 1417 (1965).

30. T. L. Chng, I. S. Orel, S. M. Starikovskaia, and I. V. Adamovich, Plasma Sources Sci. Technol. 28, 045004 (2019).

31. L. P. Babich, T. V. Loiko, and V. A. Tsukerman, Sov. Phys.-Usp. 33, 521 (1990).

32. E. I. Asinovskii, L. M. Vasilyak, and V. V. Markovets, Teplofiz. Vys. Temp. 21, 577 (1983).

33. B.-D. Huang, K. Takashima, X.-M. Zhu, and Y.-K. Pu, J. Phys. D: Appl. Phys. 48, 125202 (2015).

34. S. Eliseev, M. Timshina, A. Samokhvalov, M. Letunovskaya, A. Smirnov, K. Sergushichev, N. Kalinin, D. Belsky, and V. Burtsev, J. Phys.: Conf. Ser. 1400, 077017 (2019).

35. W. J. M. Brok, J. Dijk, M. D. Bowden, J. J. A. M. van der Mullen, and G. M. W. Kroesen, J. Phys. D: Appl. Phys. 36, 1967 (2003).

36. W. J. M. Brok, M. F. Gendre, and J. J. A. M. van der Mullen, J. Phys. D: Appl. Phys. 40, 156 (2007).

37. S. M. Starikovskaia, A. Yu. Starikovskii, and D. V. Zatsepin, J. Phys. D: Appl. Phys. 31, 1118 (1998).

38. T. L. Chng, A. Brisset, P. Jeanney, S. M. Starikovskaia, I. V. Adamovich, and P. Tardiveau, Plasma Sources Sci. Technol. 28, 09LT02 (2019).

39. G. Popa, K. Ohet, and N. Dumitraşcu, J. Phys. D: Appl. Phys. 22, 1327 (1989).

40. W. Uyterhoeven and K. W. Hess, Elektrische Gasentladungslampen (Springer, Berlin, 1938).

41. M. Steenbeck and G. Mierdel, Z. Phys. 106, 311 (1937).

42. G. E. Spivak and E. L. Stolyarova, Zh. Tekh. Fiz. 18, 279 (1948).

43. G. E. Spivak, E. L. Stolyarova, and A. I. Krokhina, Vestn. Mosk. Gos. Univ., No. 5, 14 (1949).

44. G. E. Spivak and E. L. Stolyarova, Zh. Tekh. Fiz. 20, 501 (1950).

45. K. H. Kingdom and H. E. Tanis, Phys. Rev. 53, 128 (1938).

46. L. N. Tunitskii and A. I. Ignashkov, Svetotekhnika, No. 2, 23 (1955).

PLASMA PHYSICS REPORTS Vol.46 No. $10 \quad 2020$
47. L. N. Tunitskii and V. P. Cherkasov, Sov. Phys.-Tech. Phys. 14, 1640 (1970).

48. A. V. Nedospasov and A. E. Novik, Sov. Phys.-Tech. Phys. 5, 1261 (1961).

49. N. I. Vinokurov, V. A. Gerasimov, V. V. Zaponchkovskii, and Yu. F. Fomenko, Sov. Phys.-Tech. Phys. 22, 1457 (1977).

50. V. P. Abramov and I. P. Mazan'ko, Sov. Phys.-Tech. Phys. 25, 446 (1980).

51. V. P. Abramov, P. I. Ishchenko, and I. P. Mazan'ko, Sov. Phys.-Tech. Phys. 25, 449 (1980).

52. A. Dengra, M. A. Hernandez, and V. Colomer, Contrib. Plasma Phys. 27, 283 (1987).

53. A. Dengra, J. Ballesteros, M. A. Hernandez, and V. Colomer, J. App. Phys. 68, 5507 (1990).

54. J. Ballesteros, M. A. Hernandez, A. Dengra, and V. Colomer, Contrib. Plasma Phys. 31, 595 (1991).

55. R. E. Horstman and F. M. Oude Lansink, J. Phys. D: Appl. Phys. 21, 1130 (1988).

56. M. F. Gendre, M. D. Bowden, H. Haverlag, H. C. M. van den Nieuwenhuiz, J. Gielen, and G. M. W. Kroesen, in Proceedings of Frontiers in Low Temperature Plasma Diagnostics V, Specchia, 2003, Ed. by S. De Benedics and G. Dilecce, p. 295.

57. M. F. Gendre and M. D. Bowden, H. C. M. van den Nieuwenhuizen, M. Haverlag, J. W. A. M. Gielen, and G. M. W. Kroesen, IEEE Trans. Plasma Sci. 33, 262 (2005).

58. W. J. M. Brok, M. F. Gendre, M. Haverlag, and J. J. A. M. van der Mullen, J. Phys. D: Appl. Phys. 40, 3931 (2007).

59. R. Langer, R. Garner, A. Hilscher, R. Tidecks, and S. Horn, J. Phys. D: Appl. Phys. 41, 144011 (2008).

60. M. F. Gendre, M. Haverlag, and G. M. W. Kroesen, J. Phys. D: Appl. Phys. 43, 234004 (2010).

61. R. Langer, R. Garner, I. Paul, R. Tidecks, and S. Horn, Eur. Phys. J.: Appl. Phys. 76, 10802 (2016).

62. R. Langer, I. Paul, A. Hilscher, S. Horn, and R. Tidecks, Eur. Phys. J.: Appl. Phys. 76, 30802 (2016).

63. S. A. Kalinin, A. V. Meshchanov, A. I. Shishpanov, and Yu. Z. Ionikh, Plasma Phys. Rep. 44, 345 (2018).

64. S. A. Kalinin, Candidate's Dissertation in Physics and Mathematics (St. Petersburg State University, St. Petersburg, 2019). https://disser.spbu.ru/files/2019/disser_kalinov.pdf.

65. A. I. Shishpanov, A. V. Meshchanov, S. A. Kalinin, and Y. Z. Ionikh, Plasma Sources Sci. Technol. 26, 065017 (2017).

66. A. V. Meshchanov, D. O. Ivanov, Y. Z. Ionikh, and A. I. Shishpanov, J. Phys. D: Appl. Phys. 51, 335202 (2018).

67. V. A. Lisovskii and S. D. Yakovin, Tech. Phys. 45, 727 (2000).

68. V. A. Lisovskiy, S. D. Yakovin, and V. D. Yegorenkov, J. Phys. D: Appl. Phys. 33, 2722 (2000).

69. M. M. Pejović, G. S. Ristić, and J. P. Karamarković, J. Phys. D: Appl. Phys. 35, R91 (2002).

70. A. I. Shishpanov, A. V. Meshchanov, M. D. Kuchugura, and Y. Z. Ionikh, in Proceedings of the VII Interna- 
tional Conference on Plasma Physics and Plasma Technology, Minsk, 2012, Vol. 1, p. 279.

71. A. I. Shishpanov, Yu. Z. Ionikh, and A. V. Meshchanov, Opt. Spectrosc. 120, 871 (2016).

72. L. Nie, Y. Xian, X. Lu, and K. Ostrikov, Phys. Plasmas 24, 043502 (2017).

73. M. M. Pejović and R. D. Filipović, Int. J. Electron. 67, 251 (1989).

74. V. Lj. Marković, S. R. Gocić, S. N. Stamenković, and Z. Lj. Petrović, Eur. Phys. J.: Appl. Phys. 30, 51 (2005).

75. Č. A. Maluckov and M. K. Radović, IEEE Trans. Plasma Sci. 30, 1597 (2002).

76. D. Levko, R. R. Arslanbekov, and V. I. Kolobov, Phys. Plasmas 26, 064502 (2019).

77. M. K. Radović, Č. A. Maluckov, J. P. Karamarković, S. A. Rančev, and S. D. Mitić, Rom. Rep. Phys. 66, 472 (2014).

78. Yu. Z. Ionikh, A. V. Meshchanov, and D. O. Ivanov, Tech. Phys. 64, 950 (2019).

79. H. Paetow, Z. Phys. 111, 770 (1939).

80. N. L. Aleksandrov and E. M. Bazelyan, J. Phys. D: Appl. Phys. 31, 1343 (1998).

81. S. Nijdam, G. Wormeester, E. M. van Veldhuizen, and U. Ebert, J. Phys. D: Appl. Phys. 44, 455201 (2011).

82. Y. Xia, D. Liu, W. Wang, Z. Bi, X. Wang, J. Niu, L. Ji, Y. Song, and Z. Qi, J. Phys. D: Appl. Phys. 49, 165202 (2016).

83. F. Tholin and A. Bourdon, Plasma Sources Sci.Technol. 22, 045014 (2013).

84. X. Li, R. Liu, P. Jia, K. Wu, C. Ren, and Z. Yin, Phys. Plasmas 25, 013512 (2018).

85. Milić M. Pejović, Momćilo M. Pejović, and K. Stanković, Plasma Chem. Plasma Process. 38, 415 (2018).

86. A. I. Shishpanov, Yu. Z. Ionikh, A. V. Meshchanov, and N. A. Dyatko, Plasma Phys. Rep. 40, 467 (2014).

87. A. V. Meshchanov, A. N. Korshunov, Yu. Z. Ionikh, and N. A. Dyatko, Plasma Phys. Rep. 41, 677 (2015).

88. A. V. Meshchanov, Yu. Z. Ionikh, A. I. Shishpanov, and S. A. Kalinin, Plasma Phys. Rep. 42, 978 (2016).

89. N. A. Dyatko, Yu. Z. Ionikh, A. V. Meshchanov, and A. P. Napartovich, Plasma Phys. Rep. 44, 334 (2018).

90. A. I. Shishpanov, A. V. Meshchanov, S. A. Kalinin, and Y. Z. Ionikh, in Proceedings of the 23rd Europhysics Conference on Atomic and Molecular Physics of Ionized Gases, Bratislava, 2016, p. 398.

91. Dj. A. Bošan, M. K. Radović, and Dj. M. Krompotić, J. Phys. D: Appl. Phys. 19, 2343 (1986).

92. Dj. A. Bošan, T. V. Jovanović, and Dj. M. Krompotić, J. Phys. D: Appl. Phys. 30, 3096 (1997).

93. T. V. Jovanović, Dj. A. Bošan, Dj. M. Krompotić, and M. K. Radović, J. Phys. D: Appl. Phys. 31, 3249 (1998).

94. M. M. Pejović, G. S. Ristić, and Z. Lj. Petrović, J. Phys. D: Appl. Phys. 32, 1489 (1999).

95. M. Hamamoto, Jpn. J. Appl. Phys. 45, L172 (2006).

96. M. Hamamoto, Jpn. J. Appl. Phys. 45, L175 (2006).

97. M. Hamamoto, S. Kai, T. Haizaki, and M. Ishibashi, in Proceedings of the XXVIII International Conference on Phenomena in Ionized Gases, Prague, 2007, p. 1973.
98. A. V. Meshchanov, A. I. Shishpanov, Y. Z. Ionikh, S. A. Kalinin, and N. A. Dyatko, in Proceedings of the 23rd Europhysics Conference on Atomic and Molecular Physics of Ionized Gases, Bratislava, 2016, p. 128.

99. R. I. Mints, I. I. Mil'man, and V. I. Kryuk, Sov. Phys.Usp. 19, 697 (1976).

100. Y. Gosho and A. Harada, J. Phys. D: Appl. Phys. 16, 1047 (1983).

101. O. Guaitella, I. Marinov, and A. Rousseau, Appl. Phys. Lett. 98, 071502 (2011).

102. P. F. Ambrico, M. Ambrico, L. Schiavulli, and S. De Benedictis, J. Phys. D: Appl. Phys. 47, 305201 (2014).

103. Yu. B. Golubovskii, V. A. Maiorov, J. Behnke, and J. F. Behnke, J. Phys. D: Appl. Phys. 35, 751 (2002).

104. R. Tschiersch, M. Bogaczyk, and H.-E. Wagner, J. Phys. D: Appl. Phys. 47, 365204 (2014).

105. M. Li, C. Li, H. Zhan, J. Xu, and X. Wang, Appl. Phys. Lett. 92, 031503 (2008).

106. R. Tschiersch, S. Nemschokmichal, and J. Meichsner, Plasma Sources Sci. Technol. 26, 075006 (2017).

107. S. A. Kalinin, M. A. Kapitonova, R. M. Matveev, A. V. Meshchanov, and Yu. Z. Ionikh, Plasma Phys. Rep. 44, 1009 (2018).

108. N. A. Dyatko, Yu. Z. Ionikh, A. V. Meshchanov, A. P. Napartovich, and K. A. Barzilovich, Plasma Phys. Rep. 36, 1040 (2010).

109. A. I. Shishpanov, Candidate's Dissertation in Physics and Mathematics (St. Petersburg State University, St. Petersburg, 2014). https://disser.spbu.ru/disser2/disser/dissertacia_Shishpanov.pdf.

110. S. V. Pancheshnyi, S. M. Starikovskaia, and A. Yu. Starikovskii, J. Phys. D: Appl.Phys. 32, 2219 (1999).

111. B.-D. Huang, E. Carbone, K. Takashima, X.-M. Zhu, U. Czarnetzki, and Y.-K. Pu, J. Phys. D: Appl. Phys. 51, 225202 (2018).

112. N. A. Dyatko, Yu. Z. Ionikh, S. A. Kalinin, and A. A. Mityureva, Plasma Phys. Rep. 46, 200 (2020).

113. Yu. Z. Ionikh, Yu. G. Utkin, N. V. Chernysheva, and A. S. Evdokimenko, Plasma Phys. Rep. 22, 267 (1996).

114. N. A. Dyatko, Yu. Z. Ionikh, A. V. Meshchanov, and A. P. Napartovich, Plasma Phys. Rep. 31, 871 (2005).

115. N. A. Dyatko, F. E. Latyshev, A. S. Mel'nikov, and A. P. Napartovich, Plasma Phys. Rep. 32, 158 (2006).

116. R. Kh. Amirov, E. I. Asinovskii, and V. V. Markovets, High Temp. 19, 37 (1981).

117. R. Kh. Amirov, E. I. Asinovskii, and V. V. Markovets, Plasma Phys. Rep. 27, 424 (2001).

118. N. A. Dyatko, Yu. Z. Ionikh, A. V. Meshchanov, A. P. Napartovich, and A. I. Shishpanov, Plasma Phys. Rep. 37, 505 (2011).

119. A. I. Shishpanov, D. O. Ivanov, and S. A. Kalinin, Plasma Res. Express 1, 025004 (2019).

120. E. I. Asinovsky, A. N. Lagarkov, V. V. Markovets, and I. M. Rutkevich, Plasma Sources Sci. Technol. 3, 556 (1994).

121. Z. Xiong, E. Robert, V. Sarron, J.-M. Pouvesle, and M. J. Kushner, J. Phys. D: Appl. Phys. 45, 275201 (2012).

Translated by E. Chernokozhin 